\title{
ERROR ESTIMATES FOR RAVIART-THOMAS INTERPOLATION OF ANY ORDER ON ANISOTROPIC TETRAHEDRA
}

\author{
GABRIEL ACOSTA, THOMAS APEL, RICARDO G. DURÁN, AND ARIEL L. LOMBARDI
}

\begin{abstract}
We prove optimal order error estimates for the Raviart-Thomas interpolation of arbitrary order under the maximum angle condition for triangles and under two generalizations of this condition, namely, the so-called three-dimensional maximum angle condition and the regular vertex property, for tetrahedra.

Our techniques are different from those used in previous papers on the subject, and the results obtained are more general in several aspects. First, intermediate regularity is allowed; that is, for the Raviart-Thomas interpolation of degree $k \geq 0$, we prove error estimates of order $j+1$ when the vector field being approximated has components in $W^{j+1, p}$, for triangles or tetrahedra, where $0 \leq j \leq k$ and $1 \leq p \leq \infty$. These results are new even in the two-dimensional case. Indeed, the estimate was known only in the case $j=k$. On the other hand, in the three-dimensional case, results under the maximum angle condition were known only for $k=0$.
\end{abstract}

\section{INTRODUCTION}

The Raviart-Thomas finite element spaces were introduced in 21, 23, and extended to the three-dimensional case by Nédélec [20, to approximate second order elliptic problems in mixed form. After publication of that paper there has been an increasing interest in the analysis of these spaces and on the approximation properties of the associated Raviart-Thomas interpolation operator. This interest has been motivated by the fact that, apart from the original motivation, these spaces (or rotated versions of them in the two-dimensional case) arise in several interesting applications, for example in mixed methods for plates (see [7, 8, 14]) and in the numerical approximation of fluid-structure interaction problems [5]. Also, it is well known that mixed methods are related to non-conforming methods [3, 19]; therefore, the Raviart-Thomas interpolation operator can be useful in some cases to analyze these kinds of methods (see for example [1] where a non-conforming method for the Stokes problem is analyzed).

Received by the editor September 11, 2008 and, in revised form, May 2, 2009.

2010 Mathematics Subject Classification. Primary 65N30.

Key words and phrases. Mixed finite elements, Raviart-Thomas, anisotropic finite elements.

The work of the first author was supported by Deutsche Forschungsgemeinschaft, Grant AP 72/3-1 and by Agencia Nacional de Promoción Científica y Tecnológica (ANPCyT), Argentina, Grant PAV-120.

The first, third and fourth authors were partially supported by ANPCyT, under grants PICT 2007-910, PICT 2005-33617, and PICT 2007-01307, and by Universidad de Buenos Aires, under Grant X070. 
The original error analysis developed in [20, 21, 23] is based on the so-called regularity assumption on the elements and therefore, the constants arising in the error estimates in those works depend on the ratio between the outer and inner diameter of the elements. In this way narrow or anisotropic elements, which are very important in many applications, are excluded.

For the standard Lagrange interpolation it is known, since the pioneering works 4. 17, 22] and many generalizations of them (see [2] and its references), that the regularity assumption can be relaxed to a maximum angle condition in many cases.

Error estimates for the Raviart-Thomas interpolation under conditions weaker than the regularity have been proved in several papers. In [1], the lowest order case $k=0$ was considered and optimal order error estimates were proved under the maximum angle condition for triangles and two suitable generalizations of it for tetrahedra, called the regular vertex property and the maximum angle condition. In [9], error estimates for tetrahedra in the case $k=0$ were proved under a condition which, as we will show, is equivalent to the regular vertex property. Also in [9] the authors obtained error estimates for general degree $k$ but under the assumption that the approximated vector field is divergence free. In [16], similar results were obtained for prismatic elements and functions from weighted Sobolev spaces.

It is not straightforward to extend the arguments given in [1] to higher order Raviart-Thomas approximations. In 12 it was proved that the maximum angle condition is also sufficient to obtain optimal error estimates for the case $k=1$ and $n=2$ and in [15] that result was generalized to any $k \geq 0$. Also in [15], error estimates for any $k \geq 0$ and $n=3$ were proved assuming the regular vertex property.

The error estimates obtained in [15] require "maximum regularity". To be precise, let $\Pi_{k} \mathbf{u}$ be the Raviart-Thomas interpolation of degree $k$ of $\mathbf{u}$ on a triangle $T$. Then, it was proved in [15] that

$$
\left\|\mathbf{u}-\Pi_{k} \mathbf{u}\right\|_{L^{2}(T)} \leq \frac{C}{\sin \alpha} h_{T}^{k+1}|\mathbf{u}|_{H^{k+1}(T)},
$$

where we have used the standard notation for Sobolev seminorms, $\alpha$ and $h_{T}$ are the maximum angle and the diameter of $T$ respectively, and the constant $C$ is independent of $T$. However, an estimate such as (1.1) but with $k$ replaced by $j<k$, only on the right hand side, cannot be proved by the arguments given in [15], and therefore a different approach is needed. Let us remark that this kind of estimate is important in many situations. In particular, the lowest order estimate

$$
\left\|\mathbf{u}-\Pi_{k} \mathbf{u}\right\|_{L^{2}(T)} \leq \frac{C}{\sin \alpha} h_{T}|\mathbf{u}|_{H^{1}(T)}
$$

is fundamental in the error analysis for the scalar variable in mixed approximations of second order elliptic problems. In particular the inf-sup condition can be obtained from this estimate (see for example [11, 13]).

The maximum angle condition was originally introduced for triangles. For the three-dimensional case, two different generalizations have been given. One is the Kř́žžek maximum angle condition, introduced in [18]: the angles between faces and the angles in the faces are bounded away from $\pi$. Another possible extension is the regular vertex property introduced in [1]: a family of tetrahedral elements satisfies this condition if for each element there is at least one vertex such that the unit vectors in the direction of the edges sharing that vertex are "uniformly" linearly 


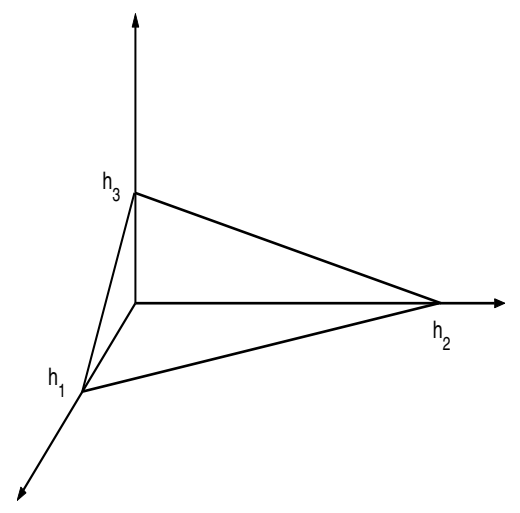

(a)

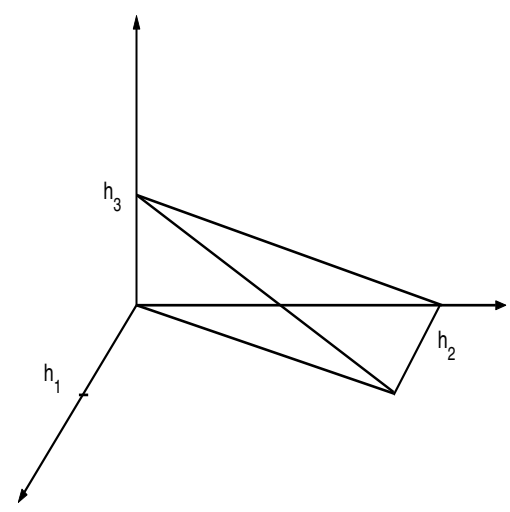

(b)

Figure 1. Two important families of elements.

independent, in the sense that the volume determined by them is uniformly bounded away from zero.

These two conditions are equivalent in two dimensions but not in three. Indeed, the Krrížek maximum angle condition allows for more general elements. This can be seen in the following way: consider the two families of elements given in Figure 1. where $h_{1}, h_{2}$ and $h_{3}$ are arbitrary positive numbers. Both families satisfy the Křižek condition, but the second family does not satisfy the regular vertex property.

Essentially these two families of elements give all possibilities. Indeed, the family of all elements satisfying the Kř́žek condition with a constant $\bar{\psi}<\pi$ (i.e., angles between faces and angles in the faces less than or equal to $\bar{\psi}$ ) can be obtained by transforming both families in the figure by "good" affine transformations (see Theorem 2.2 for the precise meaning of this). This result was obtained in 1 in the proof of Lemma 5.9. For the sake of clarity we will include this result as a theorem. On the other hand, the family of all elements satisfying the regular vertex property with a given constant (see Section 3 for the formal definition of this) is obtained by transforming in the same way only the first family in the figure. Therefore, to obtain general results under the Křížek maximum angle condition (resp., regular vertex property) it is enough to prove error estimates for both families (resp., the first family) in Figure 1 with constants independent of the relations between $h_{1}, h_{2}$ and $h_{3}$.

The error estimates in 15 for the general $\mathcal{R} T_{k}$ were obtained by assuming the regular vertex property, and the arguments given in that paper cannot be extended to treat the more general case of elements satisfying the Křržzek condition. On the other hand, as we have mentioned above, the arguments in [15 cannot be applied to obtain error estimates for functions in $H^{j+1}(T)^{n}$ with $j<k$. For these reasons we need to introduce here a different approach.

In this paper we complete the error analysis for the Raviart-Thomas interpolation of arbitrary order $k \geq 0$. We develop the analysis in the general case of $L^{p}$-based norms, generalizing also in this aspect the results of previous papers. Our arguments are different to those used in previous papers. The main point is to prove sharp estimates in reference elements. 
Let us explain the idea in the two-dimensional case. Consider the reference triangle $\widehat{T}$ which has vertices at $(0,0),(1,0)$ and $(0,1)$. A stability estimate on $\widehat{T}$ can be used to obtain the stability in a general triangle by using the Piola transform. Afterwards, error estimates can be proved combining stability with polynomial approximation results.

The original proof given in 21] uses that

$$
\left\|\Pi_{k} \mathbf{u}\right\|_{L^{2}(\widehat{T})} \leq C\|\mathbf{u}\|_{H^{1}(\widehat{T})} .
$$

In this way, the constant arising in the estimate for a general element depends on the minimum angle, and so the regularity assumption is needed. The reason for that dependence is that the complete $H^{1}$-norm appears on the right hand side. Therefore, to improve this result one may try to obtain sharper estimates on $\widehat{T}$ for each component of $\Pi_{k} \mathbf{u}$. Denote with $u_{j}$ and $\Pi_{k, j} \mathbf{u}, j=1,2$, the components of $\mathbf{u}$ and its Raviart-Thomas interpolation, respectively, and consider for example $j=1$. Ideally, we would like to have the estimate

$$
\left\|\Pi_{k, 1} \mathbf{u}\right\|_{L^{2}(\widehat{T})} \leq C\left\|u_{1}\right\|_{H^{1}(\widehat{T})} .
$$

However, an easy computation shows that if, for example, $\mathbf{u}=\left(0, x_{2}^{2}\right)$, then $\Pi_{k} \mathbf{u}=$ $\frac{1}{3}\left(x_{1}, x_{2}\right)$ and therefore the above estimate is not true. In other words, even for a rectangular triangle $\widehat{T}, \Pi_{k, 1} \mathbf{u}$ depends on both components of $\mathbf{u}$. Now, the question is: which are the essential degrees of freedom defining $\Pi_{k, 1} \mathbf{u}$ ?

To answer this question one can try to "kill" degrees of freedom by modifying $\mathbf{u}$ without changing $\Pi_{k, 1} \mathbf{u}$. A key observation is that if $\mathbf{r}=\left(0, g\left(x_{1}\right)\right)$, then $\Pi_{k, 1} \mathbf{r}=$ 0 (we will give the proof of this result for appropriate reference elements in the three-dimensional case). Therefore, if $\mathbf{v}=\left(u_{1}\left(x_{1}, x_{2}\right), u_{2}\left(x_{1}, x_{2}\right)-u_{2}\left(x_{1}, 0\right)\right)$, then $\Pi_{k, 1} \mathbf{v}=\Pi_{k, 1} \mathbf{u}$. But the normal component of $\mathbf{v}$ on the edge $\ell_{2}$ contained in the line $\left\{x_{2}=0\right\}$, i.e. $v_{2}$, vanishes, and so do all the degrees of freedom defining $\Pi_{k}$ associated with that edge. Moreover, if we now modify the second component defining $\mathbf{w}=\left(u_{1}\left(x_{1}, x_{2}\right), u_{2}\left(x_{1}, x_{2}\right)-u_{2}\left(x_{1}, 0\right)-x_{2} \alpha\right)$, for some $\alpha \in \mathcal{P}_{k-1}$, we still have that $w_{2}$ vanishes on $\ell_{2}$ and that $\Pi_{k, 1} \mathbf{w}=\Pi_{k, 1} \mathbf{u}$ (because we are modifying $\mathbf{v}$ by adding a vector field belonging to the Raviart-Thomas space of order $k$ ). But, as we will see, it is possible to choose $\alpha$ in such a way that the degrees of freedom corresponding to integrals over $\widehat{T}$ also vanish. Of course, it will be necessary to estimate some norm of $\alpha$. We will give the details of the proofs in the three-dimensional case. It is easy to see that the same arguments can be used to complete the arguments explained above for the two-dimensional case.

The new contributions of this paper can be summarized as follows:

- We prove error estimates under the maximum angle condition with order $j+1$ if the approximated function is in $W^{j+1, p}(T)^{n}, n=2,3$, where $0 \leq$ $j \leq k$ and $1 \leq p \leq \infty$.

- Under the regular vertex property we obtain estimates of anisotropic type also for general $k \geq 0$ and $1 \leq p \leq \infty$. We also show that this kind of estimate is not valid under the maximum angle condition.

Let us finally mention that the interpolation error estimates of anisotropic type are necessary when one wishes to exploit the independent element sizes $h_{1}, h_{2}$ and $h_{3}$ to treat edge singularities in elliptic problems or layers in singularly perturbed problems. The dilemma is that such estimates hold, as we show, only for tetrahedra with the regular vertex property, but it seems to be impossible to fill space by using 
these types of elements only. An anisotropic triangular prism (pentahedron) can, for example, be subdivided into three tetrahedra, from which only two satisfy the regular vertex property while the third is of the type of the element at the right hand side of Figure 1. The only known way out so far is discussed in [16. These authors use pentahedral meshes or tetrahedral meshes which are obtained by a suitable subdivision of a pentahedral mesh. Pentahedra based on a regular triangular face satisfy the regular vertex property by construction. For the approximation on tetrahedral elements they use a composition of two interpolation operators in order to avoid the above-mentioned insufficiency with the tetrahedra which do not satisfy the regular vertex property. This approach has been restricted to prismatic domains so far.

The rest of the paper is as follows. In Section 2 we introduce notation and give some preliminary results on the conditions on tetrahedra that we will work with. Then, we prove stability in $L^{p}(T)^{3}$ for the Raviart-Thomas interpolation of arbitrary degree for functions in $W^{1, p}(T)^{3}$. These stability results are proved in Section 3 for elements satisfying the regular vertex property, and in Section 4 for elements satisfying the maximum angle condition. The estimates obtained under both hypotheses are essentially different but the results are sharp. Indeed, in Section 5, we show that anisotropic type stability estimates cannot be obtained for the larger class of elements satisfying the maximum angle condition. Finally, in Section 6, we derive the error estimates from the stability results and standard approximation arguments.

\section{NotATION AND PRELIMINARY RESUltS}

In this section we recall some known results involving geometric properties of certain degenerate tetrahedra. Most of these results were proved in [1] and 2].

Given a general tetrahedron $T \subset \mathbb{R}^{3}$, $\mathbf{p}_{0}$ will denote an arbitrary vertex and, for $1 \leq i \leq 3, \ell_{i}$, with $\left\|\ell_{i}\right\|=1$, will be the directions of the edges sharing $\mathbf{p}_{0}$ and $h_{i}$ the lengths of those edges. In other words, $T$ is the convex hull of $\left\{\mathbf{p}_{0}\right\} \cup\left\{\mathbf{p}_{0}+h_{i} \ell_{i}\right\}_{1 \leq i \leq 3}$.

We will use the standard notation for Sobolev spaces $W^{k, p}(\Omega)$ of functions with all their derivatives up to the order $k$ belonging to $L^{p}(\Omega)$, denoting by $\|\cdot\|_{W^{k, p}(\Omega)}$ the associated norm. The same notation will be used for the norm of vector fields $\mathbf{u} \in W^{k, p}(\Omega)^{3}$. As is usual, we use boldface fonts for vector fields.

With $\mathcal{P}_{k}(T)$ we denote the set of polynomials of degree less than or equal to $k$ defined over $T \subset \mathbb{R}^{3}$. The Raviart-Thomas space of order $k$ is defined as

$$
\mathcal{R} \mathcal{T}_{k}=\mathcal{P}_{k}(T)^{3}+\left(x_{1}, x_{2}, x_{3}\right) \mathcal{P}_{k}(T)
$$

and for $\mathbf{u} \in W^{1, p}(T)^{3}$ the Raviart-Thomas interpolation of order $k$ is defined as $\Pi_{k} \mathbf{u} \in \mathcal{R} \mathcal{T}_{k}$ such that

$$
\begin{array}{rlrl}
\int_{F} \Pi_{k} \mathbf{u} \cdot \mathbf{n} p_{k} & =\int_{F} \mathbf{u} \cdot \mathbf{n} p_{k} & \forall p_{k} \in \mathcal{P}_{k}(F), \quad F \text { a face of } T, \\
\int_{T} \Pi_{k} \mathbf{u} \cdot \mathbf{p}_{k-1}=\int_{T} \mathbf{u} \cdot \mathbf{p}_{k-1} & \forall \mathbf{p}_{k-1} \in \mathcal{P}_{k-1}(T)^{3} .
\end{array}
$$

In the rest of the paper the letter $C$ will denote a generic constant that may change from line to line.

Now we introduce the different conditions on the elements that we will use. The first one, called the "regular vertex property", was introduced in [1]. 
Definition 2.1. A tetrahedron $T$ satisfies the "regular vertex property" with a constant $\bar{c}>0$ (written $R V P(\bar{c})$ ) if $T$ has a vertex $\mathbf{p}_{0}$, such that if $M$ is the matrix made up of $\ell_{i}, 1 \leq i \leq 3$, as columns, then $|\operatorname{det} M|>\bar{c}$.

One can easily check that a regular family of tetrahedra (with the usual definition of regularity given for example in [10]) verifies the regular vertex property. On the other hand, simple examples such as that at the left hand side of Figure1 1 show that arbitrarily narrow elements are allowed in the class given by $R V P(\bar{c})$ for a fixed $\bar{c}$.

Despite the presence of anisotropic elements, the regular vertex property arises as a natural geometric condition if one looks for Raviart-Thomas interpolation error bounds. Indeed, looking at the vertex placed at $\mathbf{p}_{0}$, one can see that the family of elements satisfying $R V P(\bar{c})$ has three normal vectors (those normals to the faces sharing $\mathbf{p}_{0}$ ) that are uniformly linearly independent (see [1] for more details), a reasonable condition, since the moments of the normal components of vector fields are used as degrees of freedom in the Raviart-Thomas interpolation. Strikingly, as was shown in [1, the uniform independence of the normal components can be somehow relaxed and error estimates valid uniformly for a wider class of elements can still be obtained for $\Pi_{0}$ (and for $\Pi_{k}$ as we will show). More precisely, we will prove error estimates under the maximum angle condition defined below, which was introduced by Krrížek in 18 and is weaker than the RVP.

Definition 2.2. A tetrahedron $T$ satisfies the "maximum angle condition" with a constant $\bar{\psi}<\pi$ (written $M A C(\bar{\psi})$ ) if the maximum angle between faces and the maximum angle inside the faces are less than or equal to $\bar{\psi}$.

Let us mention that the estimates obtained under RVP are stronger than those valid under MAC. Indeed, in the first case, the estimates are of anisotropic type (roughly speaking, this means that the estimates are given in terms of sizes in different directions and their corresponding derivatives). On the other hand, we will show that these kinds of estimates are not valid for the more general class of elements verifying the $M A C$ condition.

The definition of the maximum angle condition is strongly geometric. In order to find an equivalent condition, more appropriate for our further computations, we introduce the following definitions. In what follows, $\mathbf{e}_{i}$ will denote the canonical vectors.

Definition 2.3. A tetrahedron $T$ belongs to the family $\mathcal{F}_{1}$ if its vertices are at $\mathbf{0}$, $h_{1} \mathbf{e}_{1}, h_{2} \mathbf{e}_{2}$ and $h_{3} \mathbf{e}_{3}$, where $h_{i}>0$ are arbitrary lengths (see Figure 1a).

Definition 2.4. A tetrahedron $T$ belongs to the family $\mathcal{F}_{2}$ if its vertices are at $\mathbf{0}$, $h_{1} \mathbf{e}_{1}+h_{2} \mathbf{e}_{2}, h_{2} \mathbf{e}_{2}$ and $h_{3} \mathbf{e}_{3}$, where $h_{i}>0$ are arbitrary lengths (see Figure 1b). $\bar{c}$.

Note that elements in $\mathcal{F}_{2}$ satisfy $M A C\left(\frac{\pi}{2}\right)$ but they do not fulfill $R V P(\bar{c})$ for any

Lemma 2.1. Let $T$ be a tetrahedron satisfying $M A C(\bar{\psi})$. Then we have

(1) If $\alpha \leq \beta \leq \gamma$ are the angles of an arbitrary face of $T$, then $\gamma \geq \frac{\pi}{3}$ and $\beta, \gamma \in[(\pi-\bar{\psi}) / 2, \bar{\psi}]$.

(2) If $\mathbf{p}_{0}$ is an arbitrary vertex of $T$ and $\chi \leq \psi \leq \phi$ are the angles between faces passing through $\mathbf{p}_{0}$, then $\phi \geq \frac{\pi}{3}$ and $\psi, \phi \in[(\pi-\bar{\psi}) / 2, \bar{\psi}]$.

Proof. See 18 . 
For a matrix $M \in \mathbb{R}^{3 \times 3},\|M\|$ will denote its infinity norm. The arguments used in the following theorem are essentially contained in the proof of Theorem 7 in [18, page 516]. We include some details here for the sake of clarity.

Theorem 2.2. Let $T$ be a tetrahedron satisfying $M A C(\bar{\psi})$. Then there exists an element $\widetilde{T} \in \mathcal{F}_{1} \cup \mathcal{F}_{2}$ that can be mapped onto $T$ through an affine transformation $F(\widetilde{\mathbf{x}})=M \widetilde{\mathbf{x}}+\mathbf{c}$ with $\|M\|,\left\|M^{-1}\right\| \leq C$, where the constant $C$ depends only on $\bar{\psi}$.

Proof. Given a tetrahedron $T$ we denote with $\mathbf{p}_{i}, i=0,1,2,3$, its vertices and use the obvious notation for its faces and edges. Let $\mathbf{p}_{0} \mathbf{p}_{1} \mathbf{p}_{2}$ be an arbitrary face of $T$ and $\mathbf{p}_{3}$ its opposite vertex. We can assume that the maximum angle $\gamma$ of the face $\mathbf{p}_{0} \mathbf{p}_{1} \mathbf{p}_{2}$ is at the vertex $\mathbf{p}_{0}$. Then from Lemma 2.1 we have

$$
\sin \gamma \geq m:=\min \left\{\sin \frac{\pi-\bar{\psi}}{2}, \sin \bar{\psi}\right\}
$$

Let $\mathbf{t}_{\mathbf{1}}$ and $\mathbf{t}_{\mathbf{2}}$ be unit vectors along the edges $\mathbf{p}_{0} \mathbf{p}_{1}$ and $\mathbf{p}_{0} \mathbf{p}_{2}$. We can also assume that the angle $\omega$ between the faces $\mathbf{p}_{0} \mathbf{p}_{1} \mathbf{p}_{2}$ and $\mathbf{p}_{0} \mathbf{p}_{1} \mathbf{p}_{3}$ is not less than the angle between $\mathbf{p}_{0} \mathbf{p}_{1} \mathbf{p}_{2}$ and $\mathbf{p}_{0} \mathbf{p}_{2} \mathbf{p}_{3}$ (otherwise we interchange the notation between the vertices $\mathbf{p}_{1}$ and $\mathbf{p}_{2}$ ). Then, again from Lemma 2.1, we have

$$
\sin \omega \geq m \text {. }
$$

Now consider the triangle $\mathbf{p}_{0} \mathbf{p}_{1} \mathbf{p}_{3}$ and choose $k \in\{0,1\}$ so that the angle $\xi$ at the vertex $\mathbf{p}_{k}$ is not less than that at the vertex $\mathbf{p}_{1-k}$. Using again Lemma 2.1] we obtain

$$
\sin \xi \geq m
$$

We now take $\mathbf{t}_{\mathbf{3}}$ as the unit vector along $\mathbf{p}_{k} \mathbf{p}_{3}$ and define $M_{0}$ as the matrix made up with $\mathbf{t}_{\mathbf{1}}, \mathbf{t}_{\mathbf{2}}$ and $\mathbf{t}_{\mathbf{3}}$ as its columns. Since the columns of $M_{0}$ are unitary vectors, we have $\left\|M_{0}\right\| \leq 3$. Also, the adjugate matrix of $M_{0}$ has coefficients with absolute value bounded by 2 and therefore, $\left\|M_{0}^{-1}\right\| \leq 6 /\left|\operatorname{det} M_{0}\right|$. Then, to obtain the desired bound for $\left\|M_{0}^{-1}\right\|$ it is enough to show that $\left|\operatorname{det} M_{0}\right|$ is bounded below by a constant which depends only on $\bar{\psi}$.

Consider the parallelepiped generated by the vectors $\mathbf{t}_{\mathbf{1}}, \mathbf{t}_{\mathbf{2}}$ and $\mathbf{t}_{\mathbf{3}}$. Let $z$ be its height in the direction perpendicular to $\mathbf{t}_{\mathbf{1}}$ and $\mathbf{t}_{\mathbf{2}}$ and $y$ the height of the face generated by $\mathbf{t}_{\mathbf{1}}$ and $\mathbf{t}_{\mathbf{3}}$ in the direction perpendicular to $\mathbf{t}_{\mathbf{1}}$.

Since $\left\|\mathbf{t}_{\mathbf{i}}\right\|=1$ we have

$$
\left|\operatorname{det} M_{0}\right|=z \sin \gamma=y \sin \omega \sin \gamma=\sin \xi \sin \omega \sin \gamma \geq m^{3}
$$

as we wanted to prove.

Obviously, the same properties are satisfied by the matrix $M_{1}$ made up of $\mathbf{t}_{\mathbf{2}},-\mathbf{t}_{\mathbf{1}}$ and $\mathbf{t}_{\mathbf{3}}$ as its columns.

Now, if $k=0$, define $h_{1}=\left|\mathbf{p}_{0} \mathbf{p}_{1}\right|, h_{2}=\left|\mathbf{p}_{0} \mathbf{p}_{2}\right|$ and $h_{3}=\left|\mathbf{p}_{0} \mathbf{p}_{3}\right|$ and take $\widetilde{T} \in \mathcal{F}_{1}$ with vertices at $\mathbf{0}, h_{1} \mathbf{e}_{1}, h_{2} \mathbf{e}_{2}$ and $h_{3} \mathbf{e}_{3}$; while if $k=1$, define $h_{1}=\left|\mathbf{p}_{0} \mathbf{p}_{2}\right|$, $h_{2}=\left|\mathbf{p}_{0} \mathbf{p}_{1}\right|$ and $h_{3}=\left|\mathbf{p}_{1} \mathbf{p}_{3}\right|$ and take $\widetilde{T} \in \mathcal{F}_{2}$ with vertices at $\mathbf{0}, h_{1} \mathbf{e}_{1}+h_{2} \mathbf{e}_{2}, h_{2} \mathbf{e}_{2}$ and $h_{3} \mathbf{e}_{3}$. Then, for any case, we have that $\widetilde{\mathbf{x}} \mapsto M_{k} \widetilde{\mathbf{x}}+\mathbf{p}_{k}$ maps $\widetilde{T}$ onto $T$.

As mentioned above, the regular vertex property is stronger than the maximum angle condition. Indeed, the following theorem shows that, under $\operatorname{RVP}(\bar{c})$, the reference family in the previous theorem can be restricted to $\mathcal{F}_{1}$. 
Theorem 2.3. Let $T$ be a tetrahedron satisfying $R V P(\bar{c})$. Then, there exists an element $\widetilde{T} \in \mathcal{F}_{1}$ that can be mapped onto $T$ through an affine transformation $F(\widetilde{\mathbf{x}})=M \widetilde{\mathbf{x}}+\mathbf{p}_{0}$ with $\|M\|,\left\|M^{-1}\right\| \leq C$, where the constant $C$ depends only on $\bar{c}$. Furthermore, if $h_{i}, i=1,2,3$ are the lengths of the edges of $T$ sharing the vertex $\mathbf{p}_{0}$, we can take $\widetilde{T} \in \mathcal{F}_{1}$ such that, for $i=1,2,3, h_{i}$ is the length in the direction $\mathbf{e}_{i}$.

Proof. Let $\mathbf{p}_{0}$ and $\ell_{i}$ be as in the definition of $R V P(\bar{c})$ and $h_{i}$ be the length of the edge of $T$ with direction $\ell_{i}$.

Take $M$ as the matrix made up with $\ell_{i}$ as its columns. Since $|\operatorname{det}(M)|>\bar{c}$ and $\ell_{i}$ are unitary vectors, then it is easy to check that $\|M\| \leq C$ and $\left\|M^{-1}\right\| \leq C$ with a constant $C$ depending only on a lower bound of $|\operatorname{det}(M)|$ and therefore on $\bar{c}$.

Then, if $\widetilde{T}$ is the tetrahedron of $\mathcal{F}_{1}$ with lengths $h_{i}$ in the directions $\mathbf{e}_{i}$, the affine transformation $F(\widetilde{\mathbf{x}})=M \widetilde{\mathbf{x}}+\mathbf{p}_{\mathbf{0}}$ maps $\widetilde{T}$ onto $T$.

Remark 2.1. It is not difficult to see that the converses of Theorems 2.2 and 2.3 hold true. Namely, the family of elements obtained by transforming $\mathcal{F}_{1} \cup \mathcal{F}_{2}$ (resp. $\mathcal{F}_{1}$ ) by affine maps $\widetilde{\mathbf{x}} \mapsto M \widetilde{\mathbf{x}}+\mathbf{c}$, where $\|M\|,\left\|M^{-1}\right\| \leq C$, satisfies $M A C(\bar{\psi}$ ) (resp. $R V P(\bar{c})$ ) for some $\bar{\psi}$ (resp. $\bar{c}$ ) which depends only on $C$.

Let us mention that error estimates for the Raviart-Thomas interpolation were obtained also in [9] by Buffa, Costabel and Dauge. In the next section we will compare the results in 9] with ours. In order to do that we recall here their assumption on the elements. Let $\widehat{T}$ be the tetrahedron with vertices at $(0,0,0)$, $(1,0,0),(0,1,0)$ and $(0,0,1)$.

Definition 2.5. A tetrahedron $T$ satisfies the condition $B C D$ with a constant $\bar{c}_{1}$ (written $B C D\left(\bar{c}_{1}\right)$ ), if there exists an affine transformation $F_{T}$ mapping $\widehat{T}$ onto $T$ with the following property:

There exists a local Cartesian system associated to $T$ such that, if $\widehat{\mathbf{x}}$ are the canonical coordinates of a point in $\widehat{T}$ and $\mathbf{x}_{T}$ are the coordinates of $F_{T}(\widehat{\mathbf{x}})$ in the local system, then

$$
\mathbf{x}_{T}=A \widehat{\mathbf{x}}+\mathbf{p}_{0}
$$

for some $\mathbf{p}_{0} \in \mathbb{R}^{3}$ and a matrix $A \in \mathbb{R}^{3 \times 3}$ which can be written as $A=B D=D \breve{B}$ with $\|B\|,\left\|B^{-1}\right\|,\|\breve{B}\|,\left\|\breve{B}^{-1}\right\| \leq \bar{c}_{1}$.

We will show by the following two lemmata that the $B C D$ condition is equivalent to the $R V P$.

Lemma 2.4. If an element $T$ satisfies $B C D\left(\bar{c}_{1}\right)$, then it satisfies $R V P(\bar{c})$ for a constant $\bar{c}$ depending only on $\bar{c}_{1}$.

Proof. With the notation introduced in Definition 2.5, and denoting by $\mathrm{x}$ the coordinates of $F_{T}(\widehat{\mathbf{x}})$ in the canonical system, we have $\mathbf{x}=C \mathbf{x}_{T}$, where $C$ is an orthogonal matrix. Therefore, $\mathbf{x}=C B D \widehat{\mathbf{x}}+C \mathbf{p}_{0}$ with $\|B\|,\left\|B^{-1}\right\| \leq \bar{c}_{1}$. Observing that $\widetilde{\mathbf{x}}=D \widehat{\mathbf{x}}$ transforms $\widehat{T}$ into a tetrahedron in the family $\mathcal{F}_{1}$ we conclude that the map $C B \widetilde{\mathbf{x}}+C \mathbf{p}_{0}$ satisfies the properties of the map in Remark 2.1. Consequently, $T$ satisfies the regular vertex property with a constant depending only on $\bar{c}_{1}$.

Lemma 2.5. If an element $T$ satisfies $R V P(\bar{c})$, then it satisfies $B C D\left(\bar{c}_{1}\right)$ for a constant $\bar{c}_{1}$ depending only on $\bar{c}$. 
Proof. Suppose that $T$ satisfies $R V P(\bar{c})$. According to Theorem 2.3 there exists a transformation mapping $\widehat{T}$ onto $T$ which, in canonical global coordinates $\widehat{\mathbf{x}}$ and $\mathbf{x}$, can be written as $\mathbf{x}=M D \widehat{\mathbf{x}}+\mathbf{p}_{0}$ with $\|M\|,\left\|M^{-1}\right\| \leq C$ and $D=\operatorname{diag}\left(h_{1}, h_{2}, h_{3}\right)$. Moreover, we can assume without loss of generality that $h_{1} \geq h_{2} \geq h_{3}$.

Now using the $Q R$-decomposition $M=Q R$, where $Q$ is an orthogonal matrix and $R$ is upper triangular, we obtain $\mathbf{x}=Q R D \widehat{\mathbf{x}}+\mathbf{p}_{0}$. Therefore, if we introduce the local coordinates $\mathbf{x}_{T}=Q^{T} \mathbf{x}$ we have $\mathbf{x}_{T}=R D \widehat{\mathbf{x}}+Q^{T} \mathbf{p}_{0}$.

Let us check that this local coordinate system satisfies the conditions from Definition 2.5. It is easy to see that $B:=R$ has the desired properties since $\|B\|=\|R\|=\left\|Q^{T} M\right\| \leq\|Q\|\|M\| \leq\|M\| \leq C$ and $\left\|B^{-1}\right\|=\left\|R^{-1}\right\|=\left\|M^{-1} Q\right\| \leq$ $\left\|M^{-1}\right\|\|Q\| \leq C$.

On the other hand, if $B D=D \breve{B}$ we have $\breve{B}=D^{-1} R D$. Then, the entries of $\breve{B}$ are given by $\breve{b}_{i j}=r_{i j} h_{j} / h_{i}$ if $j \geq i$, and $b_{i j}=0$ if $j<i$. Since $h_{1} \geq h_{2} \geq h_{3}$ and $\|R\| \leq C$ we have $\max _{i j}\left|\breve{b}_{i j}\right| \leq \max _{i j}\left|r_{i j}\right| \leq C$, i.e. $\|\breve{B}\| \leq C$. In the same way we obtain the boundedness of $\breve{B}^{-1}=D^{-1} R^{-1} D$ from the boundedness of $R^{-1}$.

\section{Stability under the Regular Vertex PROPERTy}

The goal of this section is to prove the stability in $L^{p}$ for the Raviart-Thomas interpolation of arbitrary order of functions in $W^{1, p}(T)^{3}$, for families of elements satisfying the regular vertex property. Throughout this section, the reference element $\widehat{T}$ is defined as the tetrahedron with vertices at $(0,0,0),(1,0,0),(0,1,0)$ and $(0,0,1)$.

The main result of this section is the following theorem.

Theorem 3.1. Let $k \geq 0$ and $T$ be a tetrahedron satisfying $R V P(\bar{c})$. If $\mathbf{p}_{0}$ is the regular vertex, $\ell_{i}, i=1,2,3$ are unitary vectors with the directions of the edges sharing $\mathbf{p}_{0}, h_{i}, i=1,2,3$, the lengths of these edges, and $h_{T}$ the diameter of $T$, then there exists a constant $C$ depending only on $k$ and $\bar{c}$ such that, for all $\mathbf{u} \in W^{1, p}(T)^{3}$, $1 \leq p \leq \infty$,

$$
\left\|\Pi_{k} \mathbf{u}\right\|_{L^{p}(T)} \leq C\left(\|\mathbf{u}\|_{L^{p}(T)}+\sum_{i, j} h_{j}\left\|\frac{\partial u_{i}}{\partial \ell_{j}}\right\|_{L^{p}(T)}+h_{T}\|\operatorname{div} \mathbf{u}\|_{L^{p}(T)}\right) .
$$

Let us mention that a similar result was proved in [9]. There the authors assume the condition $B C D$ mentioned in the previous section (see Definition 2.5). As we have seen in Lemmata 2.4 and 2.5. this assumption is equivalent to the $\operatorname{RVP}(\bar{c})$. Nevertheless, our result is stronger since the estimates given in $[9]$ for general degree $k$ were proved only for divergence free vector fields $\mathbf{u}$.

Coming back to our error analysis let us mention that Theorem 3.1 will follow from Theorem 2.3 once we have proved error estimates for elements in the family $\mathcal{F}_{1}$.

First we will prove appropriate estimates in the reference element $\widehat{T}$. This is the object of the next two lemmas. Afterwards, estimates for elements in $\mathcal{F}_{1}$ will be obtained by scaling arguments.

We denote with $\widehat{F}_{i}$ the face of $\widehat{T}$ normal to $\mathbf{n}_{i}$, with $\mathbf{n}_{1}=(-1,0,0), \mathbf{n}_{2}=$ $(0,-1,0), \mathbf{n}_{3}=(0,0,-1)$ and $\mathbf{n}_{4}=\frac{1}{\sqrt{3}}(1,1,1)$. We will use the same notation for a function of two variables than for its extension to $\widehat{T}$ as a function independent of the other variable, for example, $f\left(x_{2}, x_{3}\right)$ will denote a function defined on $\widehat{F}_{1}$ as 
well as one defined in $\widehat{T}$ (anyway, the meaning in each case will be clear from the context). In the same way, the same notation will be used to denote a polynomial $p_{k}$ on a face and a polynomial in three variables such that its restriction to that face agrees with $p_{k}$. For example, for $p_{k} \in \mathcal{P}_{k}\left(\widehat{F}_{4}\right)$ we will write $p_{k}\left(1-x_{2}-x_{3}, x_{2}, x_{3}\right)$. In what follows, $\widehat{\Pi}_{k, i} \mathbf{u}$ denotes the $i$-th component of $\widehat{\Pi}_{k} \mathbf{u}$.

Lemma 3.2. Let $f \in L^{p}\left(\widehat{F}_{1}\right), g \in L^{p}\left(\widehat{F}_{2}\right)$, and $h \in L^{p}\left(\widehat{F}_{3}\right)$. If

$$
\mathbf{u}\left(x_{1}, x_{2}, x_{3}\right)=\left(f\left(x_{2}, x_{3}\right), 0,0\right), \quad \mathbf{v}\left(x_{1}, x_{2}, x_{3}\right)=\left(0, g\left(x_{1}, x_{3}\right), 0\right),
$$

and

$$
\mathbf{w}\left(x_{1}, x_{2}, x_{3}\right)=\left(0,0, h\left(x_{1}, x_{2}\right)\right),
$$

then their Raviart-Thomas interpolations are of the same form; namely, there exist $q_{i} \in \mathcal{P}_{k}\left(\widehat{F}_{i}\right), i=1,2,3$, such that

$$
\widehat{\Pi}_{k} \mathbf{u}=\left(q_{1}\left(x_{2}, x_{3}\right), 0,0\right), \quad \widehat{\Pi}_{k} \mathbf{v}=\left(0, q_{2}\left(x_{1}, x_{3}\right), 0\right),
$$

and

$$
\widehat{\Pi}_{k} \mathbf{w}=\left(0,0, q_{3}\left(x_{1}, x_{2}\right)\right) .
$$

Proof. Let us prove for example the first equality; the other two are obviously analogous. Since $\operatorname{div} \mathbf{u}=0$, we have that $\operatorname{div} \widehat{\Pi}_{k} \mathbf{u}=0$ and therefore, from a wellknown property of the Raviart-Thomas interpolation (see for example [7, 13]), it follows that $\widehat{\Pi}_{k} \mathbf{u} \in \mathcal{P}_{k}(\widehat{T})^{3}$.

On the other hand, now using (2.1) for $i=2,3$, and that $u_{2}=u_{3}=0$, we have

$$
\int_{\widehat{F}_{i}} \widehat{\Pi}_{k, i} \mathbf{u} p_{k}=0 \quad \forall p_{k} \in \mathcal{P}_{k}\left(F_{i}\right), \quad i=2,3,
$$

and then, taking $p_{k}=\widehat{\Pi}_{k, i} \mathbf{u}$, we conclude that $\left.\widehat{\Pi}_{k, i} \mathbf{u}\right|_{\widehat{F}_{i}}=0$ for $i=2,3$. Therefore $\widehat{\Pi}_{k, i} \mathbf{u}=x_{i} r_{i}$ for some $r_{i} \in \mathcal{P}_{k-1}(\widehat{T})$ and so, now using (2.2) and again that $u_{2}=$ $u_{3}=0$, we obtain that, for $i=2,3, \widehat{\Pi}_{k, i} \mathbf{u}=0$ in $\widehat{T}$ as we wanted to show.

Finally, since $\operatorname{div} \widehat{\Pi}_{k} \mathbf{u}=0$, it follows that $\frac{\partial \widehat{\Pi}_{k, 1} \mathbf{u}}{\partial x_{1}}=0$ and so, $\widehat{\Pi}_{k, 1} \mathbf{u}$ is independent of $x_{1}$.

Lemma 3.3. There exists a constant $C$ depending only on $k$ such that, for all $\mathbf{u}=\left(u_{1}, u_{2}, u_{3}\right) \in W^{1, p}(\widehat{T})^{3}$,

$$
\left\|\widehat{\Pi}_{k, i} \mathbf{u}\right\|_{L^{p}(\widehat{T})} \leq C\left(\left\|u_{i}\right\|_{W^{1, p}(\widehat{T})}+\|\operatorname{div} \mathbf{u}\|_{L^{p}(\widehat{T})}\right), \quad i=1,2,3 .
$$

Proof. From the previous lemma we know that, if

$$
\mathbf{v}=\left(u_{1}, u_{2}-u_{2}\left(x_{1}, 0, x_{3}\right), u_{3}-u_{3}\left(x_{1}, x_{2}, 0\right)\right),
$$

then $\widehat{\Pi}_{k, 1} \mathbf{u}=\widehat{\Pi}_{k, 1} \mathbf{v}$.

Let $\alpha, \beta \in \mathcal{P}_{k-1}(\widehat{T})$ be such that

$$
\int_{\widehat{T}}\left(v_{2}-x_{2} \alpha\right) p_{k-1}=0 \quad \text { and } \quad \int_{\widehat{T}}\left(v_{3}-x_{3} \beta\right) p_{k-1}=0 \quad \forall p_{k-1} \in \mathcal{P}_{k-1}(\widehat{T}) .
$$

Observe that those $\alpha$ and $\beta$ exist. Indeed, it is easy to prove uniqueness (and therefore existence) of the solution of the square linear systems of equations defining them.

Now define $\mathbf{w}=\left(v_{1}, v_{2}-x_{2} \alpha, v_{3}-x_{3} \beta\right)$. Since $\left(0, x_{2} \alpha, x_{3} \beta\right) \in \mathcal{R} T_{k}(\widehat{T})$ it follows that $\widehat{\Pi}_{k, 1} \mathbf{v}=\widehat{\Pi}_{k, 1} \mathbf{w}$ and therefore $\widehat{\Pi}_{k, 1} \mathbf{u}=\widehat{\Pi}_{k, 1} \mathbf{w}$. 
Taking into account that $\left.w_{2}\right|_{\widehat{F}_{2}}=0$ and $\left.w_{3}\right|_{\widehat{F}_{3}}=0$ and the equations (3.2), it follows that $\widehat{\Pi} \mathbf{w}$ is determined by the equations

$$
\begin{aligned}
\int_{\widehat{T}} \widehat{\Pi}_{k, 1} \mathbf{w} p_{k-1} & =\int_{\widehat{T}} w_{1} p_{k-1} \quad \forall p_{k-1} \in \mathcal{P}_{k-1}(\widehat{T}), \\
\int_{\widehat{T}} \widehat{\Pi}_{k, 2} \mathbf{w} p_{k-1} & =0 \quad \forall p_{k-1} \in \mathcal{P}_{k-1}(\widehat{T}), \\
\int_{\widehat{T}} \widehat{\Pi}_{k, 3} \mathbf{w} p_{k-1} & =0 \quad \forall p_{k-1} \in \mathcal{P}_{k-1}(\widehat{T}), \\
\int_{\widehat{F}_{1}} \widehat{\Pi}_{k, 1} \mathbf{w} p_{k} & =\int_{\widehat{F}_{1}} w_{1} p_{k} \quad \forall p_{k} \in \mathcal{P}_{k}\left(\widehat{F}_{1}\right), \\
\int_{\widehat{F}_{2}} \widehat{\Pi}_{k, 2} \mathbf{w} p_{k} & =0 \quad \forall p_{k} \in \mathcal{P}_{k}\left(\widehat{F}_{2}\right), \\
\int_{\widehat{F}_{3}} \widehat{\Pi}_{k, 3} \mathbf{w} p_{k} & =0 \quad \forall p_{k} \in \mathcal{P}_{k}\left(\widehat{F}_{3}\right), \\
\int_{\widehat{F}_{4}}\left(\widehat{\Pi}_{k, 1} \mathbf{w}+\widehat{\Pi}_{k, 2} \mathbf{w}+\widehat{\Pi}_{k, 3} \mathbf{w}\right) p_{k} & =\int_{\widehat{F}_{4}}\left(w_{1}+w_{2}+w_{3}\right) p_{k} \quad \forall p_{k} \in \mathcal{P}_{k}\left(\widehat{F}_{4}\right) .
\end{aligned}
$$

Now, for $p_{k} \in \mathcal{P}_{k}(\widehat{T})$, we have

$$
\begin{aligned}
\int_{\widehat{T}} \operatorname{div} \mathbf{w} p_{k} & =-\int_{\widehat{T}} \mathbf{w} \cdot \nabla p_{k}+\int_{\partial \widehat{T}} \mathbf{w} \cdot \mathbf{n} p_{k}, \\
& =-\int_{\widehat{T}} \mathbf{w} \cdot \nabla p_{k}+\frac{1}{\sqrt{3}} \int_{\widehat{F}_{4}}\left(w_{1}+w_{2}+w_{3}\right) p_{k}+\int_{\partial \widehat{T} \backslash \widehat{F}_{4}} \mathbf{w} \cdot \mathbf{n} p_{k},
\end{aligned}
$$

but, from the definition of $\mathbf{w}$, we have

$$
-\int_{\widehat{T}} \mathbf{w} \cdot \nabla p_{k}=-\int_{\widehat{T}} w_{1} \frac{\partial p_{k}}{\partial x_{1}} \quad \text { and } \quad \int_{\partial \widehat{T} \backslash \widehat{F}_{4}} \mathbf{w} \cdot \mathbf{n} p_{k}=-\int_{\widehat{F}_{1}} w_{1} p_{k} .
$$

Therefore, for all $p_{k} \in \mathcal{P}_{k}(\widehat{T})$,

$$
\frac{1}{\sqrt{3}} \int_{\widehat{F}_{4}}\left(w_{1}+w_{2}+w_{3}\right) p_{k}=\int_{\widehat{T}} \operatorname{div} \mathbf{w} p_{k}+\int_{\widehat{T}} w_{1} \frac{\partial p_{k}}{\partial x_{1}}+\int_{\widehat{F}_{1}} w_{1} p_{k} .
$$

But,

$$
\operatorname{div} \mathbf{w}=\operatorname{div} \mathbf{v}-\operatorname{div}\left(0, x_{2} \alpha, x_{3} \beta\right)=\operatorname{div} \mathbf{u}-\operatorname{div}\left(0, x_{2} \alpha, x_{3} \beta\right) .
$$

So, using (3.4), (3.3), and standard arguments, we obtain

$$
\begin{aligned}
& \left\|\widehat{\Pi}_{k, 1} \mathbf{u}\right\|_{L^{p}(\widehat{T})}=\left\|\widehat{\Pi}_{k, 1} \mathbf{w}\right\|_{L^{p}(\widehat{T})} \\
& \quad \leq C\left(\left\|u_{1}\right\|_{W^{1, p}(\widehat{T})}+\|\operatorname{div} \mathbf{u}\|_{L^{p}(\widehat{T})}+\left\|\operatorname{div}\left(0, x_{2} \alpha, x_{3} \beta\right)\right\|_{L^{p}(\widehat{T})}\right) .
\end{aligned}
$$

Then, to obtain (3.1) for $i=1$, it is enough to show that

$$
\left\|\operatorname{div}\left(0, x_{2} \alpha, x_{3} \beta\right)\right\|_{L^{p}(\widehat{T})} \leq C\left(\left\|u_{1}\right\|_{W^{1, p}(\widehat{T})}+\|\operatorname{div} \mathbf{u}\|_{L^{p}(\widehat{T})}\right) .
$$


For $p_{k} \in \mathcal{P}_{k}(\widehat{T})$ we have

$$
\begin{aligned}
0= & \int_{\widehat{T}}\left(0, v_{2}-x_{2} \alpha, v_{3}-x_{3} \beta\right) \cdot \nabla p_{k} \\
= & -\int_{\widehat{T}} \operatorname{div}\left(0, v_{2}-x_{2} \alpha, v_{3}-x_{3} \beta\right) p_{k} \\
& \quad+\int_{\partial \widehat{T}}\left[\left(v_{2}-x_{2} \alpha\right) n_{2}+\left(v_{3}-x_{3} \beta\right) n_{3}\right] p_{k} .
\end{aligned}
$$

Now we take $p_{k}\left(x_{1}, x_{2}, x_{3}\right)=\left(1-x_{1}-x_{2}-x_{3}\right) p_{k-1}$ with $p_{k-1} \in \mathcal{P}_{k-1}(\widehat{T})$. Then, since $p_{k}=0$ on $\widehat{F}_{4},\left(v_{2}-x_{2} \alpha\right) n_{2}=0$ on $\partial \widehat{T} \backslash F_{4}$ and $\left(v_{3}-x_{3} \beta\right) n_{3}=0$ on $\partial \widehat{T} \backslash F_{4}$, it follows that, in the last equation, the boundary integral vanishes. Then,

$$
\int_{\widehat{T}}\left(1-x_{1}-x_{2}-x_{3}\right) \operatorname{div}\left(0, v_{2}-x_{2} \alpha, v_{3}-x_{3} \beta\right) p_{k-1}=0 .
$$

That is, for all $p_{k-1} \in \mathcal{P}_{k-1}(\widehat{T})$,

$$
\int_{\widehat{T}}\left(1-x_{1}-x_{2}-x_{3}\right) \operatorname{div}\left(0, x_{2} \alpha, x_{3} \beta\right) p_{k-1}=\int_{\widehat{T}}\left(1-x_{1}-x_{2}-x_{3}\right) \operatorname{div}\left(0, v_{2}, v_{3}\right) p_{k-1} \text {. }
$$

Therefore, taking $p_{k-1}=\operatorname{div}\left(0, x_{2} \alpha, x_{3} \beta\right)$ and applying the Hölder inequality we obtain

$$
\begin{aligned}
\int_{\widehat{T}}\left(1-x_{1}-x_{2}-x_{3}\right) \mid \operatorname{div}(0 & \left., x_{2} \alpha, x_{3} \beta\right)\left.\right|^{2} \\
& \leq C\left\|\operatorname{div}\left(0, v_{2}, v_{3}\right)\right\|_{L^{p}(\widehat{T})}\left\|\operatorname{div}\left(0, x_{2} \alpha, x_{3} \beta\right)\right\|_{L^{p^{\prime}}(\widehat{T})} .
\end{aligned}
$$

But, since all the norms on $\mathcal{P}_{k-1}(\widehat{T})$ are equivalent, we conclude that

$$
\left\|\operatorname{div}\left(0, x_{2} \alpha, x_{3} \beta\right)\right\|_{L^{p}(\widehat{T})} \leq C\left\|\operatorname{div}\left(0, v_{2}, v_{3}\right)\right\|_{L^{p}(\widehat{T})} .
$$

Now, observe that $\operatorname{div}\left(0, v_{2}, v_{3}\right)=\operatorname{div}\left(0, u_{2}, u_{3}\right)$ and

$$
\left\|\operatorname{div}\left(0, u_{2}, u_{3}\right)\right\|_{L^{p}(\widehat{T})} \leq C\left(\|\operatorname{div} \mathbf{u}\|_{L^{p}(\widehat{T})}+\left\|u_{1}\right\|_{W^{1, p}(\widehat{T})}\right) .
$$

Then, (3.5) follows from (3.6).

Clearly, the estimates for $\widehat{\Pi}_{k, 2} \mathbf{u}$ and $\widehat{\Pi}_{k, 3} \mathbf{u}$ can be proved analogously.

From the previous lemma and a change of variables we obtain estimates for elements in $\mathcal{F}_{1}$.

The Raviart-Thomas operators on the elements $\widehat{T}$ and $\widetilde{T}$ will be denoted by $\widehat{\Pi}_{k}$ and $\widetilde{\Pi}_{k}$, respectively. Analogous notation will be used for variables and derivatives or differential operators on $\widehat{T}$ and $\widetilde{T}$ whenever needed for clarity.

Proposition 3.4. Let $\widetilde{T} \in \mathcal{F}_{1}$ be the element with vertices at $\mathbf{0}, h_{1} \mathbf{e}_{1}, h_{2} \mathbf{e}_{2}$ and $h_{3} \mathbf{e}_{3}$, where $h_{i}>0$. There exists a constant $C$ depending only on $k$ such that, for $\widetilde{\mathbf{u}}=\left(\tilde{u}_{1}, \tilde{u}_{2}, \tilde{u}_{3}\right) \in W^{1, p}(\widetilde{T})^{3}$ and $i=1,2,3$,

$$
\left\|\widetilde{\Pi}_{k, i} \widetilde{\mathbf{u}}\right\|_{L^{p}(\widetilde{T})} \leq C\left(\left\|\tilde{u}_{i}\right\|_{L^{p}(\widetilde{T})}+\sum_{j=1}^{3} h_{j}\left\|\frac{\partial \tilde{u}_{i}}{\partial \tilde{x}_{j}}\right\|_{L^{p}(\widetilde{T})}+h_{i}\|\widetilde{\operatorname{div} \widetilde{\mathbf{u}}}\|_{L^{p}(\widetilde{T})}\right) .
$$


Proof. Let $\widehat{\mathbf{u}} \in W^{1, p}(\widehat{T})^{3}$ be defined via the Piola transform by

$$
\widetilde{\mathbf{u}}(\widetilde{\mathbf{x}})=\frac{1}{\operatorname{det} B} B \widehat{\mathbf{u}}(\widehat{\mathbf{x}}), \quad \widetilde{\mathbf{x}}=B \widehat{\mathbf{x}}, \quad \text { with } B=\left(\begin{array}{ccc}
h_{1} & 0 & 0 \\
0 & h_{2} & 0 \\
0 & 0 & h_{3}
\end{array}\right) .
$$

It is known that (see for example [13, 21])

$$
\widetilde{\Pi}_{k} \widetilde{\mathbf{u}}(\widetilde{\mathbf{x}})=\frac{1}{\operatorname{det} B} B \widehat{\Pi}_{k} \widehat{\mathbf{u}}(\widehat{\mathbf{x}})
$$

and

$$
\widetilde{\operatorname{div}} \widetilde{\mathbf{u}}(\widetilde{\mathbf{x}})=\frac{1}{\operatorname{det} B} \widehat{\operatorname{div}} \widehat{\mathbf{u}}(\widehat{\mathbf{x}}) .
$$

Consider for example $i=1$ (the other cases are of course analogous). Using (3.1) we have

$$
\begin{aligned}
\left\|\widetilde{\Pi}_{k, 1} \widetilde{\mathbf{u}}\right\|_{L^{p}(\widetilde{T})}^{p} & =\frac{h_{1} h_{2} h_{3}}{h_{2}^{p} h_{3}^{p}}\left\|\widehat{\Pi}_{k, 1} \widehat{\mathbf{u}}\right\|_{L^{p}(\widehat{T})}^{p} \\
& \leq C \frac{h_{1} h_{2} h_{3}}{h_{2}^{p} h_{3}^{p}}\left(\left\|\hat{u}_{1}\right\|_{W^{1, p}(\widehat{T})}^{p}+\|\widehat{\operatorname{div} \widehat{\mathbf{u}}}\|_{L^{p}(\widehat{T})}^{p}\right) \\
& \leq C\left(\left\|\tilde{u}_{1}\right\|_{L^{p}(\widetilde{T})}^{p}+\sum_{j=1}^{3} h_{j}^{p}\left\|\frac{\partial \tilde{u}_{1}}{\partial \tilde{x}_{j}}\right\|_{L^{p}(\widetilde{T})}^{p}+h_{1}^{p} \| \widetilde{\left.\operatorname{div} \widetilde{\mathbf{u}} \|_{L^{p}(\widetilde{T})}^{p}\right)}\right.
\end{aligned}
$$

as we wanted to show.

We are finally ready to prove the main theorem of this section.

Proof of Theorem 3.1 . To simplify notation we assume $\mathbf{p}_{0}=\mathbf{0}$. From Theorem 2.3 we know that, if $\widetilde{T} \in \mathcal{F}_{1}$ is the element with vertices at $\mathbf{0}, h_{1} \mathbf{e}_{1}, h_{2} \mathbf{e}_{2}$ and $h_{3} \mathbf{e}_{3}$, there exists a matrix $M$ such that the associated linear transformation maps $\widetilde{T}$ onto T. Moreover, $M \mathbf{e}_{\mathbf{i}}=\ell_{i}, i=1,2,3$.

Now, given $\mathbf{u} \in W^{1, p}(T)^{3}$ we define $\widetilde{\mathbf{u}} \in W^{1, p}(\widetilde{T})^{3}$ via the Piola transform; namely,

$$
\mathbf{u}(\mathbf{x})=\frac{1}{\operatorname{det} M} M \widetilde{\mathbf{u}}(\widetilde{\mathbf{x}}), \quad \mathbf{x}=M \widetilde{\mathbf{x}}
$$

Using Proposition 3.4 after the change of variables $\mathbf{x} \mapsto \widetilde{\mathbf{x}}$ we have

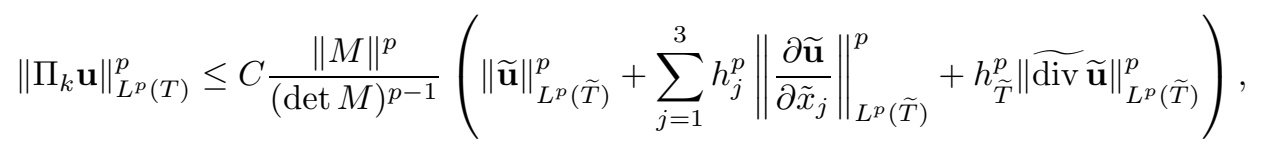

where $h_{\widetilde{T}}$ is the diameter of $\widetilde{T}$ and $\frac{\partial \widetilde{\mathbf{u}}}{\partial \tilde{x}_{j}}$ denotes the vector $\left(\frac{\partial \tilde{u}_{1}}{\partial \tilde{x}_{j}}, \frac{\partial \tilde{u}_{2}}{\partial \tilde{x}_{j}}, \frac{\partial \tilde{u}_{3}}{\partial \tilde{x}_{j}}\right)^{t}$. But,

$$
\frac{\partial \widetilde{\mathbf{u}}}{\partial \tilde{x}_{j}}=\operatorname{det} M M^{-1} \frac{\partial \mathbf{u}}{\partial \ell_{j}}, \quad \operatorname{div} \mathbf{u}(\mathbf{x})=\frac{1}{\operatorname{det} M} \widetilde{\operatorname{div}} \widetilde{\mathbf{u}}(\widetilde{\mathbf{x}}),
$$

and $h_{\widetilde{T}} \leq\left\|M^{-1}\right\| h_{T}$. Therefore we arrive at

$$
\left\|\Pi_{k} \mathbf{u}\right\|_{L^{p}(T)}^{p} \leq C\|M\|^{p}\left\|M^{-1}\right\|^{p}\left(\|\mathbf{u}\|_{L^{p}(T)}^{p}+\sum_{j=1}^{3} h_{j}^{p}\left\|\frac{\partial \mathbf{u}}{\partial \ell_{j}}\right\|_{L^{p}(T)}^{p}+h_{T}^{p}\|\operatorname{div} \mathbf{u}\|_{L^{p}(T)}^{p}\right)
$$

and recalling that $\|M\|,\left\|M^{-1}\right\| \leq C$ with $C$ depending only on $\bar{c}$, we conclude the proof. 


\section{Stability under the maximum Angle CONDition}

In this section we prove a stability result weaker than that obtained in the previous section but which is valid for families of elements satisfying the maximum angle condition.

The estimate obtained here, although uniform in the class of elements satisfying $\operatorname{MAC}(\bar{\psi})$, is weaker than the estimate obtained in Theorem 3.1 under the stronger $R V P(\bar{c})$ hypothesis. Indeed, in front of each derivative, the diameter $h_{T}$ appears instead of the length of the edge in the direction of the derivative. However, our result is optimal. In fact, we will show in the next section that estimates such as those in Theorem 3.1 are not valid in general under the maximum angle condition.

The main result of this section is the following theorem.

Theorem 4.1. Let $k \geq 0$ and let $T$ be a tetrahedron with diameter $h_{T}$ satisfying $\operatorname{MAC}(\bar{\psi})$. There exists a constant $C$ depending only on $k$ and $\bar{\psi}$ such that, for all $\mathbf{u} \in W^{1, p}(T)^{3}, 1 \leq p \leq \infty$,

$$
\left\|\Pi_{k} \mathbf{u}\right\|_{L^{p}(T)} \leq C\left(\|\mathbf{u}\|_{L^{p}(T)}+h_{T}\|\nabla \mathbf{u}\|_{L^{p}(T)}\right) .
$$

The steps to prove this theorem are similar to those followed in Section 3. Now our reference element $\widehat{T}$ is the tetrahedron with vertices at $\mathbf{0}, \mathbf{e}_{1}+\mathbf{e}_{2}, \mathbf{e}_{2}$ and $\mathbf{e}_{3}$. For $\mathbf{n}_{1}=(1,0,0), \mathbf{n}_{2}=\frac{1}{\sqrt{2}}(1,-1,0), \mathbf{n}_{3}=(0,0,1)$ and $\mathbf{n}_{4}=\frac{1}{\sqrt{2}}(0,1,1)$ we denote with $\widehat{F}_{i}$ the face of $\widehat{T}$ normal to $\mathbf{n}_{i}$ and with $\bar{F}_{2}$ the projection of $\widehat{F}_{2}$ onto the plane given by $x_{2}=0$.

Lemma 4.2. Let $f \in L^{p}\left(\widehat{F}_{1}\right), g \in L^{p}\left(\bar{F}_{2}\right)$, and $h \in L^{p}\left(\widehat{F}_{3}\right)$. If

$$
\mathbf{u}\left(x_{1}, x_{2}, x_{3}\right)=\left(f\left(x_{2}, x_{3}\right), 0,0\right), \quad \mathbf{v}\left(x_{1}, x_{2}, x_{3}\right)=\left(0, g\left(x_{1}, x_{3}\right), 0\right),
$$

and

$$
\mathbf{w}\left(x_{1}, x_{2}, x_{3}\right)=\left(0,0, h\left(x_{1}, x_{2}\right)\right),
$$

then their Raviart-Thomas interpolations are of the same form; namely, there exist $q_{i} \in \mathcal{P}_{k}\left(\widehat{F}_{i}\right), i=1,3$, and $q_{2} \in \mathcal{P}_{k}\left(\bar{F}_{2}\right)$ such that

$$
\widehat{\Pi}_{k} \mathbf{u}=\left(q_{1}\left(x_{2}, x_{3}\right), 0,0\right), \quad \widehat{\Pi}_{k} \mathbf{v}=\left(0, q_{2}\left(x_{1}, x_{3}\right), 0\right),
$$

and

$$
\widehat{\Pi}_{k} \mathbf{w}=\left(0,0, q_{3}\left(x_{1}, x_{2}\right)\right)
$$

Proof. The proof is similar to that of Lemma 3.2. We will prove the first equality; the other two follow in an analogous way.

First, we have that $\operatorname{div} \widehat{\Pi}_{k} \mathbf{u}=0$ and therefore $\widehat{\Pi}_{k} \mathbf{u} \in \mathcal{P}_{k}(\widehat{T})^{3}$. Then, proceeding exactly as in the proof of Lemma 3.2 we obtain that $\widehat{\Pi}_{k, 3} \mathbf{u}=0$ in $\widehat{T}$. Analogously, now using (2.1) for $i=4$ we have $\left.\left(\widehat{\Pi}_{k, 2} \mathbf{u}+\widehat{\Pi}_{k, 3} \mathbf{u}\right)\right|_{\widehat{F}_{4}}=0$, and so

$$
\widehat{\Pi}_{k, 2} \mathbf{u}+\widehat{\Pi}_{k, 3} \mathbf{u}=\left(1-x_{2}-x_{3}\right) r
$$

for some $r \in \mathcal{P}_{k-1}(\widehat{T})$. Consequently, now using (2.2) and that $u_{2}=u_{3}=0$, we obtain $\widehat{\Pi}_{k, 2} \mathbf{u}+\widehat{\Pi}_{k, 3} \mathbf{u}=0$ in $\widehat{T}$. Then, since we already know that $\widehat{\Pi}_{k, 3} \mathbf{u}=0$, we conclude that $\widehat{\Pi}_{k, 2} \mathbf{u}=0$ in $\widehat{T}$.

Therefore, $\widehat{\Pi}_{k} \mathbf{u}=(q, 0,0)$ for some $q \in \mathcal{P}_{k}(\widehat{T})$ but, since $\operatorname{div} \widehat{\Pi}_{k} \mathbf{u}=0$, it follows that $\widehat{\Pi}_{k, 1}$ is independent of $x_{1}$. 
Lemma 4.3. There exists a constant $C_{1}$ depending only on $k$ such that, for all $\mathbf{u}=\left(u_{1}, u_{2}, u_{3}\right) \in W^{1, p}(\widehat{T})^{3}$,

$$
\begin{aligned}
& \left\|\widehat{\Pi}_{k, 1} \mathbf{u}\right\|_{L^{2}(\widehat{T})} \leq C_{1}\left(\left\|u_{1}\right\|_{W^{1, p}(\widehat{T})}+\|\operatorname{div} \mathbf{u}\|_{L^{p}(\widehat{T})}\right) \\
& \left\|\widehat{\Pi}_{k, 2} \mathbf{u}\right\|_{L^{2}(\widehat{T})} \leq C_{1}\left(\left\|u_{2}\right\|_{W^{1, p}(\widehat{T})}+\left\|\frac{\partial u_{1}}{\partial x_{1}}\right\|_{L^{p}(\widehat{T})}+\left\|\frac{\partial u_{3}}{\partial x_{3}}\right\|_{L^{p}(\widehat{T})}\right) \\
& \left\|\widehat{\Pi}_{k, 3} \mathbf{u}\right\|_{L^{p}(\widehat{T})} \leq C_{1}\left(\left\|u_{3}\right\|_{W^{1, p}(\widehat{T})}+\|\operatorname{div} \mathbf{u}\|_{L^{p}(\widehat{T})}\right) .
\end{aligned}
$$

In particular, for $i=1,2,3$,

$$
\left\|\widehat{\Pi}_{k, i} \mathbf{u}\right\|_{L^{2}(\widehat{T})} \leq C_{2}\left(\left\|u_{i}\right\|_{W^{1, p}(\widehat{T})}+\sum_{\substack{j=1 \\ j \neq i}}^{3}\left\|\frac{\partial u_{j}}{\partial x_{j}}\right\|_{L^{p}(\widehat{T})}\right)
$$

for another constant $C_{2}$ which depends only on $k$.

Proof. Let $\mathbf{v}=\left(u_{1}, u_{2}-u_{2}\left(x_{1}, x_{1}, x_{3}\right), u_{3}-u_{3}\left(x_{1}, x_{2}, 0\right)\right)$ and $\alpha, \beta \in \mathcal{P}_{k-1}(\widehat{T})$ such that

$$
\int_{\widehat{T}}\left(v_{2}-\left(x_{1}-x_{2}\right) \alpha\right) p_{k-1}=0 \quad \text { and } \quad \int_{\widehat{T}}\left(v_{3}-x_{3} \beta\right) p_{k-1}=0 \quad \forall p_{k-1} \in \mathcal{P}_{k-1}(\widehat{T}),
$$

and define

$$
\mathbf{w}=\left(u_{1}, u_{2}-u_{2}\left(x_{1}, x_{1}, x_{3}\right)-\left(x_{1}-x_{2}\right) \alpha, u_{3}-u_{3}\left(x_{1}, x_{2}, 0\right)-x_{3} \beta\right) .
$$

Then, since $\left(0,\left(x_{1}-x_{2}\right) \alpha, x_{3} \beta\right) \in \mathcal{R} T_{k}$, it follows from Lemma 4.2 that

$$
\widehat{\Pi}_{k, 1} \mathbf{u}=\widehat{\Pi}_{k, 1} \mathbf{w} .
$$

Now, taking into account the definition of $\mathbf{w}$ and (4.6), we have that $\widehat{\Pi}_{k} \mathbf{w}$ is defined by

$$
\begin{aligned}
\int_{\widehat{T}} \widehat{\Pi}_{k, 1} \mathbf{w} p_{k-1} & =\int_{\widehat{T}} w_{1} p_{k-1} \quad \forall p_{k-1} \in \mathcal{P}_{k-1}(\widehat{T}), \\
\int_{\widehat{T}} \widehat{\Pi}_{k, 2} \mathbf{w} p_{k-1} & =0 \quad \forall p_{k-1} \in \mathcal{P}_{k-1}(\widehat{T}), \\
\int_{\widehat{T}} \widehat{\Pi}_{k, 3} \mathbf{w} p_{k-1} & =0 \quad \forall p_{k-1} \in \mathcal{P}_{k-1}(\widehat{T}), \\
\int_{\widehat{F}_{1}} \widehat{\Pi}_{k, 1} \mathbf{w} p_{k} & =\int_{\widehat{F}_{1}} w_{1} p_{k} \quad \forall p_{k} \in \mathcal{P}_{k}\left(\widehat{F}_{1}\right), \\
\int_{\widehat{F}_{2}}\left(\widehat{\Pi}_{k, 1} \mathbf{w}-\widehat{\Pi}_{k, 2} \mathbf{w}\right) p_{k} & =\int_{\widehat{F}_{2}} w_{1} p_{k} \quad \forall p_{k} \in \mathcal{P}_{k}\left(\widehat{F}_{2}\right), \\
\int_{\widehat{F}_{3}} \widehat{\Pi}_{k, 3} \mathbf{w} p_{k} & =0 \\
\int_{\widehat{F}_{4}}\left(\widehat{\Pi}_{k, 2} \mathbf{w}+\widehat{\Pi}_{k, 3} \mathbf{w}\right) p_{k} & =\int_{\widehat{F}_{4}}\left(w_{2}+w_{3}\right) p_{k} \quad \forall p_{k} \in \mathcal{P}_{k}\left(\widehat{F}_{3}\right),
\end{aligned}
$$


But, using again (4.6) we have, for $p_{k} \in \mathcal{P}_{k}(\widehat{T})$,

$$
\begin{aligned}
\int_{\widehat{T}} \operatorname{div}\left(0, w_{2}, w_{3}\right) p_{k} & =-\int_{\widehat{T}}\left(0, w_{2}, w_{3}\right) \cdot \nabla p_{k} \\
& +\int_{\partial \widehat{T} \backslash \widehat{F}_{4}}\left(w_{2} n_{2}+w_{3} n_{3}\right) p_{k}+\frac{1}{\sqrt{2}} \int_{\widehat{F}_{4}}\left(w_{2}+w_{3}\right) p_{k} \\
& =\frac{1}{\sqrt{2}} \int_{\widehat{F}_{4}}\left(w_{2}+w_{3}\right) p_{k} .
\end{aligned}
$$

Then, it follows from (4.7) and (4.8) that

$$
\begin{aligned}
\left\|\widehat{\Pi}_{1, k} \mathbf{w}\right\|_{L^{p}(\widehat{T})} \leq & C\left(\left\|w_{1}\right\|_{W^{1, p}(\widehat{T})}+\left\|\operatorname{div}\left(0, w_{2}, w_{3}\right)\right\|_{L^{p}(\widehat{T})}\right) \\
\leq & C\left(\left\|u_{1}\right\|_{W^{1, p}(\widehat{T})}+\|\operatorname{div} \mathbf{u}\|_{L^{p}(\widehat{T})}\right. \\
& \left.\quad+\left\|\operatorname{div}\left(0,\left(x_{1}-x_{2}\right) \alpha, x_{3} \beta\right)\right\|_{L^{p}(\widehat{T})}\right) .
\end{aligned}
$$

Therefore, to conclude the proof of (4.2), it is enough to show that

$$
\left\|\operatorname{div}\left(0,\left(x_{1}-x_{2}\right) \alpha, x_{3} \beta\right)\right\|_{L^{p}(\widehat{T})} \leq C\left(\left\|u_{1}\right\|_{W^{1, p}(\widehat{T})}+\|\operatorname{div} \mathbf{u}\|_{L^{p}(\widehat{T})}\right) .
$$

But, for all $p_{k} \in \mathcal{P}_{k}(\widehat{T})$, we have

$$
0=\int_{\widehat{T}}\left(0, w_{2}, w_{3}\right) \cdot \nabla p_{k}=-\int_{\widehat{T}} \operatorname{div}\left(0, w_{2}, w_{3}\right)+\int_{\partial \widehat{T}}\left(w_{2} n_{2}+w_{3} n_{3}\right) p_{k} .
$$

Now, taking $p_{k}=\left(1-x_{2}-x_{3}\right) p_{k-1}$ with $p_{k-1} \in \mathcal{P}_{k-1}(\widehat{T})$ the boundary integral in the last equation vanishes, and therefore we obtain

$$
\int_{\widehat{T}}\left(1-x_{2}-x_{3}\right) \operatorname{div}\left(0,\left(x_{1}-x_{2}\right) \alpha, x_{3} \beta\right) p_{k-1}=\int_{\widehat{T}}\left(1-x_{2}-x_{3}\right) \operatorname{div}\left(0, u_{2}, u_{3}\right) p_{k-1} .
$$

Then, (4.9) can be obtained with an argument such as that used for (3.5). Clearly, the proof of inequality (4.4) is analogous to that of (4.2).

Now, to prove (4.3), take $\mathbf{v}=\left(u_{1}-u_{1}\left(0, x_{2}, x_{3}\right), u_{2}, u_{3}-u_{3}\left(x_{1}, x_{2}, 0\right)\right), \alpha, \beta \in$ $\mathcal{P}_{k-1}(\widehat{T})$ such that

$$
\int_{\widehat{T}}\left(v_{1}-x_{1} \alpha\right) p_{k-1}=0 \quad \text { and } \quad \int_{\widehat{T}}\left(v_{3}-x_{3} \beta\right) p_{k-1}=0 \quad \forall p_{k-1} \in \mathcal{P}_{k-1}(\widehat{T}),
$$

and define

$$
\mathbf{w}=\left(v_{1}-x_{1} \alpha, v_{2}, v_{3}-x_{3} \beta\right) .
$$

Using again Lemma 4.2 and that $\left(x_{1} \alpha, 0, x_{3} \beta\right) \in \mathcal{R} T_{k}$ we obtain

$$
\widehat{\Pi}_{k, 2} \mathbf{u}=\widehat{\Pi}_{k, 2} \mathbf{w}
$$


In this case, it follows from the definition of $\mathbf{w}$ that $\Pi_{k} \mathbf{w}$ is defined by

$$
\begin{aligned}
\int_{\widehat{T}} \widehat{\Pi}_{k, 1} \mathbf{w} p_{k-1} & =0 \quad \forall p_{k-1} \in \mathcal{P}_{k-1}(\widehat{T}), \\
\int_{\widehat{T}} \widehat{\Pi}_{k, 2} \mathbf{w} p_{k-1} & =\int_{\widehat{T}} w_{2} p_{k-1} \quad \forall p_{k-1} \in \mathcal{P}_{k-1}(\widehat{T}), \\
\int_{\widehat{T}} \widehat{\Pi}_{k, 3} \mathbf{w} p_{k-1} & =0 \quad \forall p_{k-1} \in \mathcal{P}_{k-1}(\widehat{T}), \\
\int_{F_{1}} \widehat{\Pi}_{k, 1} \mathbf{w} p_{k} & =0 \quad \forall p_{k} \in \mathcal{P}_{k}\left(\widehat{F}_{1}\right), \\
\int_{\widehat{F}_{2}}\left(\widehat{\Pi}_{k, 1} \mathbf{w}-\widehat{\Pi}_{k, 2} \mathbf{w}\right) p_{k} & =\int_{F_{2}}\left(w_{1}-w_{2}\right) p_{k} \quad \forall p_{k} \in \mathcal{P}_{k}\left(\widehat{F}_{2}\right), \\
\int_{\widehat{F}_{3}} \Pi_{k, 3} \mathbf{w} p_{k} & =0 \quad \forall p_{k} \in \mathcal{P}_{k}\left(\widehat{F}_{3}\right), \\
\int_{\widehat{F}_{4}}\left(\widehat{\Pi}_{k, 2} \mathbf{w}+\widehat{\Pi}_{k, 3} \mathbf{w}\right) p_{k} & =\int_{\widehat{F}_{4}}\left(w_{2}+w_{3}\right) p_{k} \quad \forall p_{k} \in \mathcal{P}_{k}\left(\widehat{F}_{4}\right) .
\end{aligned}
$$

But, it is easy to check by integration by parts that, for all $p_{k} \in \mathcal{P}_{k}(\widehat{T})$,

$$
\int_{\widehat{T}} \operatorname{div}\left(0, w_{2}, w_{3}\right) p_{k}=-\int_{\widehat{T}} w_{2} \frac{\partial p_{k}}{\partial x_{2}}-\frac{1}{\sqrt{2}} \int_{\widehat{F}_{2}} w_{2} p_{k}+\frac{1}{\sqrt{2}} \int_{\widehat{F}_{4}}\left(w_{2}+w_{3}\right) p_{k}
$$

and

$$
\int_{\widehat{T}} \operatorname{div}\left(w_{1}, w_{2}, 0\right) p_{k}=-\int_{\widehat{T}} w_{2} \frac{\partial p_{k}}{\partial x_{2}}+\frac{1}{\sqrt{2}} \int_{\widehat{F}_{4}} w_{2} p_{k}+\frac{1}{\sqrt{2}} \int_{\widehat{F}_{2}}\left(w_{1}-w_{2}\right) p_{k} .
$$

Now, it follows from (4.10), (4.11) and (4.12) that

$$
\left\|\widehat{\Pi}_{2, k} \mathbf{w}\right\|_{L^{p}(\widehat{T})} \leq C\left(\left\|w_{2}\right\|_{W^{1, p}(\widehat{T})}+\left\|\operatorname{div}\left(0, w_{2}, w_{3}\right)\right\|_{L^{p}(\widehat{T})}+\left\|\operatorname{div}\left(w_{1}, w_{2}, 0\right)\right\|_{L^{p}(\widehat{T})}\right)
$$

and therefore, using the definition of $\mathbf{w}$, we obtain

$$
\begin{array}{r}
\left\|\widehat{\Pi}_{2, k} \mathbf{w}\right\|_{L^{p}(\widehat{T})} \leq C\left(\left\|u_{2}\right\|_{W^{1, p}(\widehat{T})}+\left\|\frac{\partial u_{1}}{\partial x_{1}}\right\|_{L^{p}(\widehat{T})}\right. \\
\left.+\left\|\frac{\partial u_{3}}{\partial x_{3}}\right\|_{L^{p}(\widehat{T})}+\left\|\frac{\partial\left(x_{1} \alpha\right)}{\partial x_{1}}\right\|_{L^{p}(\widehat{T})}+\left\|\frac{\partial\left(x_{3} \beta\right)}{\partial x_{3}}\right\|_{L^{p}(\widehat{T})}\right) .
\end{array}
$$

Then, to conclude the proof of (4.3) we have to estimate the last two terms in the above inequality. From the definition of $w_{3}$ we have, for all $p_{k} \in \mathcal{P}_{k}(\widehat{T})$,

$$
0=\int_{\widehat{T}} w_{3} \frac{\partial p_{k}}{\partial x_{3}}=-\int_{\widehat{T}} \frac{\partial w_{3}}{\partial x_{3}} p_{k}+\int_{\partial \widehat{T}} w_{3} n_{3} p_{k}
$$

but, if we take $p_{k}=\left(1-x_{2}-x_{3}\right) p_{k-1}$ with $p_{k-1} \in \mathcal{P}_{k-1}(\widehat{T})$ the boundary integral in the last equation vanishes, and therefore

$$
\int_{\widehat{T}}\left(1-x_{2}-x_{3}\right) \frac{\partial\left(x_{3} \beta\right)}{\partial x_{3}} p_{k-1}=\int_{\widehat{T}}\left(1-x_{2}-x_{3}\right) \frac{\partial u_{3}}{\partial x_{3}} p_{k-1} \quad \forall p_{k-1} \in \mathcal{P}_{k-1}(\widehat{T}),
$$

from which we obtain

$$
\left\|\frac{\partial\left(x_{3} \beta\right)}{\partial x_{3}}\right\|_{L^{p}(\widehat{T})} \leq C\left\|\frac{\partial u_{3}}{\partial x_{3}}\right\|_{L^{p}(\widehat{T})} .
$$


In a similar way we can prove

$$
\left\|\frac{\partial\left(x_{1} \alpha\right)}{\partial x_{1}}\right\|_{L^{p}(\widehat{T})} \leq C\left\|\frac{\partial u_{1}}{\partial x_{1}}\right\|_{L^{p}(\widehat{T})}
$$

and so (4.3) is proved.

Proceeding as in the previous section we now obtain estimates for elements in $\mathcal{F}_{2}$.

Proposition 4.4. Let $\widetilde{T} \in \mathcal{F}_{2}$ be the element with vertices at $\mathbf{0}, h_{1} \mathbf{e}_{1}+h_{2} \mathbf{e}_{2}, h_{2} \mathbf{e}_{2}$ and $h_{3} \mathbf{e}_{3}$, where $h_{i}>0$. There exists a constant $C$ depending only on $k$ such that, for $\widetilde{\mathbf{u}}=\left(\tilde{u}_{1}, \tilde{u}_{2}, \tilde{u}_{3}\right) \in W^{1, p}(\widetilde{T})^{3}$ and $i=1,2,3$,

$$
\left\|\widetilde{\Pi}_{k, i} \widetilde{\mathbf{u}}\right\|_{L^{p}(\widetilde{T})} \leq C\left(\left\|\tilde{u}_{i}\right\|_{L^{p}(\widetilde{T})}+\sum_{j=1}^{3} h_{j}\left\|\frac{\partial \tilde{u}_{i}}{\partial \tilde{x}_{j}}\right\|_{L^{p}(\widetilde{T})}+h_{i} \sum_{\substack{j=1 \\ j \neq i}}^{3}\left\|\frac{\partial \tilde{u}_{j}}{\partial \tilde{x}_{j}}\right\|_{L^{p}(\widetilde{T})}\right)
$$

Proof. We proceed as in the proof of Proposition 3.4. Recall that now our reference element $\widehat{T}$ is the tetrahedron with vertices at $\mathbf{0}, \mathbf{e}_{1}+\mathbf{e}_{2}, \mathbf{e}_{2}$ and $\mathbf{e}_{3}$. Therefore, the same linear map given by $B$ in Proposition 3.4 maps $\widehat{T}$ in $\widetilde{T}$. Then, if $\widehat{\mathbf{u}} \in W^{1, p}(\widehat{T})^{3}$ is defined via the Piola transform we have

$$
\widetilde{\Pi}_{k} \widetilde{\mathbf{u}}(\widetilde{\mathbf{x}})=\frac{1}{\operatorname{det} B} B \widehat{\Pi}_{k} \widehat{\mathbf{u}}(\widehat{\mathbf{x}})
$$

and

$$
\widetilde{\operatorname{div}} \widetilde{\mathbf{u}}(\widetilde{\mathbf{x}})=\frac{1}{\operatorname{det} B} \widehat{\operatorname{div}} \widehat{\mathbf{u}}(\widehat{\mathbf{x}})
$$

Using (4.5) and changing variables we have

$$
\begin{aligned}
\left\|\widetilde{\Pi}_{k, i} \widetilde{\mathbf{u}}\right\|_{L^{p}(\widetilde{T})}^{p} & =\frac{h_{1} h_{2} h_{3}}{h_{2}^{p} h_{3}^{p}}\left\|\widehat{\Pi}_{k, i} \widehat{\mathbf{u}}\right\|_{L^{p}(\widehat{T})}^{p} \\
& \leq C \frac{h_{1} h_{2} h_{3}}{h_{2}^{p} h_{3}^{p}}\left(\left\|\hat{u}_{i}\right\|_{L^{p}(\widehat{T})}^{p}+\sum_{j=1}^{3}\left\|\frac{\partial \hat{u}_{i}}{\partial \hat{x}_{j}}\right\|_{L^{p}(\widehat{T})}^{p}+\sum_{\substack{j=1 \\
j \neq i}}^{3}\left\|\frac{\partial \hat{u}_{j}}{\partial \hat{x}_{j}}\right\|_{L^{p}(\widehat{T})}^{p}\right) \\
& =C\left(\left\|\tilde{u}_{i}\right\|_{L^{p}(\tilde{T})}^{p}+\sum_{j=1}^{3} h_{j}^{p}\left\|\frac{\partial \tilde{u}_{i}}{\partial \tilde{x}_{j}}\right\|_{L^{p}(\widetilde{T})}^{p}+h_{i}^{p} \sum_{\substack{j=1 \\
j \neq i}}^{3}\left\|\frac{\partial \tilde{u}_{j}}{\partial \tilde{x}_{j}}\right\|_{L^{p}(\widetilde{T})}^{p}\right)
\end{aligned}
$$

and therefore (4.13) is proved.

Remark 4.1. For $i=1$ and $i=3$ a better result can be obtained. Indeed, by the same arguments used in the previous proposition, but now using (4.2) and (4.4), we can prove the following estimates:

$$
\left\|\widetilde{\Pi}_{k, i} \widetilde{\mathbf{u}}\right\|_{L^{p}(\widetilde{T})} \leq C\left(\left\|\tilde{u}_{i}\right\|_{L^{p}(\widetilde{T})}+\sum_{j=1}^{3} h_{j}\left\|\frac{\partial \tilde{u}_{i}}{\partial \tilde{x}_{j}}\right\|_{L^{p}(\widetilde{T})}+h_{2}\|\widetilde{\operatorname{div}} \widetilde{\mathbf{u}}\|_{L^{p}(\widetilde{T})}\right) .
$$

Anyway, this clearly depends on the particular orientation of the element and so, it does not seem to be useful for general tetrahedra.

We can now prove the main result of this section. 
Proof of Theorem 4.1, From Theorem 2.2 we know that there exists $\widetilde{T} \in \mathcal{F}_{1} \cup \mathcal{F}_{2}$ that can be mapped onto $T$ through an affine transformation $\widetilde{\mathbf{x}} \mapsto M \widetilde{\mathbf{x}}+\mathbf{c}$, with $\|M\|,\left\|M^{-1}\right\| \leq C$ for a constant $C$ depending only on $\bar{\psi}$. To simplify notation assume that $\mathbf{c}=\mathbf{0}$.

If $\widetilde{T} \in \mathcal{F}_{1}$, then $T$ satisfies the regular vertex property with a constant which depends only on $\bar{\psi}$ and so (4.1) follows immediately from Theorem 3.1. Therefore, we may assume that $\widetilde{T} \in \mathcal{F}_{2}$ and has vertices at $\mathbf{0}, h_{1} \mathbf{e}_{1}+h_{2} \mathbf{e}_{2}, h_{2} \mathbf{e}_{2}$ and $h_{3} \mathbf{e}_{3}$, where $h_{i}>0$.

Given $\mathbf{u} \in W^{1, p}(T)^{3}$ we again use the Piola transform and define $\widetilde{\mathbf{u}} \in W^{1, p}(\widetilde{T})^{3}$ given by

Then, using that

$$
\mathbf{u}(\mathbf{x})=\frac{1}{\operatorname{det} M} M \widetilde{\mathbf{u}}(\widetilde{\mathbf{x}}), \quad \mathbf{x}=M \widetilde{\mathbf{x}} .
$$

$$
\Pi_{k} \mathbf{u}(\mathbf{x})=\frac{1}{\operatorname{det} M} M \widetilde{\Pi}_{k} \widetilde{\mathbf{u}}(\widetilde{\mathbf{x}}),
$$

changing variables and using (4.1) in $\widetilde{T}$ we obtain

$$
\left\|\Pi_{k} \mathbf{u}\right\|_{L^{p}(T)}^{p} \leq C\|M\|^{p}\left\|M^{-1}\right\|^{p}\left(\|\mathbf{u}\|_{L^{p}(T)}^{p}+h_{T}^{p}\|M\|^{p}\|D \mathbf{u}\|_{L^{p}(T)}^{p}\right)
$$

concluding the proof.

\section{SharpNess OF the RESUlts}

In view of the results of the previous sections, it is natural to ask whether the estimate obtained under the maximum angle condition could be improved. The goal of this section is to show that this is not possible.

Consider the element $\widetilde{T} \in \mathcal{F}_{2}$ with vertices at $\mathbf{0}, h_{1} \mathbf{e}_{1}+h_{2} \mathbf{e}_{2}, h_{2} \mathbf{e}_{2}$ and $h_{3} \mathbf{e}_{3}$ and with diameter $h_{T}$. We are going to show that the inequality

$$
\left\|\widetilde{\Pi}_{k, 2} \widetilde{\mathbf{u}}\right\|_{L^{p}(\widetilde{T})} \leq C\left(\|\widetilde{\mathbf{u}}\|_{L^{p}(\widetilde{T})}+\sum_{i, j=1}^{3} h_{j}\left\|\frac{\partial \tilde{u}_{i}}{\partial \tilde{x}_{j}}\right\|_{L^{p}(\widetilde{T})}+h_{T}\|\widetilde{\operatorname{div} \widetilde{\mathbf{u}}}\|_{L^{p}(\widetilde{T})}\right),
$$

with a constant $C$ independent of $h_{1}, h_{2}$ and $h_{3}$, does not hold for some $\widetilde{\mathbf{u}}=$ $\left(\tilde{u}_{1}, \tilde{u}_{2}, \tilde{u}_{3}\right) \in W^{1, p}(\widetilde{T})^{3}$.

Suppose that (5.11), with $C$ independent of $h_{1}, h_{2}$ and $h_{3}$, holds true for all $\widetilde{\mathbf{u}} \in W^{1, p}(\widetilde{T})^{3}$. Let $\widehat{T}$ be the reference element used in Section 4 i.e., $\widehat{T}$ has vertices at $\mathbf{0}, \mathbf{e}_{1}+\mathbf{e}_{2}, \mathbf{e}_{2}$ and $\mathbf{e}_{3}$. Then, with $\widehat{\mathbf{u}} \in W^{1, p}(\widehat{T})^{3}$ we associate $\widetilde{\mathbf{u}} \in W^{1, p}(\widetilde{T})^{3}$ defined via the Piola transform with the linear transformation used in the proof of Theorem 4.1 .

To simplify notation we drop the hat from now on and write $\mathbf{u}$ instead of $\widehat{\mathbf{u}}$ and write $x_{i}$ for the variables in $\widehat{T}$.

A simple computation shows that from inequality (5.1) we obtain

$$
\left\|\Pi_{k, 2} \mathbf{u}\right\|_{L^{p}(\widehat{T})} \leq C \frac{1}{h_{2}}\left(\sum_{i=1}^{3} h_{i}\left\|\mathbf{u}_{i}\right\|_{W^{1, p}(\widehat{T})}+h_{T}\|\operatorname{div} \mathbf{u}\|_{L^{p}(\widehat{T})}\right) .
$$

Then, taking $h_{1}=h_{3}=h_{2}^{2}$ (with $h_{2}<1$ ), we would have

$$
\begin{aligned}
& \left\|\Pi_{k, 2} \mathbf{u}\right\|_{L^{p}(\widehat{T})} \\
& \quad \leq C\left(h_{2}\left\|u_{1}\right\|_{W^{1, p}(\widehat{T})}+\left\|u_{2}\right\|_{W^{1, p}(\widehat{T})}+h_{2}\left\|u_{3}\right\|_{W^{1, p}(\widehat{T})}+\|\operatorname{div} \mathbf{u}\|_{L^{p}(\widehat{T})}\right),
\end{aligned}
$$


and letting $h_{2} \rightarrow 0$ we would arrive at

$$
\left\|\Pi_{k, 2} \mathbf{u}\right\|_{L^{p}(\widehat{T})} \leq C\left(\left\|u_{2}\right\|_{W^{1, p}(\widehat{T})}+\|\operatorname{div} \mathbf{u}\|_{L^{p}(\widehat{T})}\right) .
$$

However, we are going to show that there exists $\mathbf{u} \in W^{1, p}(\widehat{T})^{3}$ for which inequality (5.2) is not valid. In fact, in the next proposition we will give, for each $k \geq 0$, a function $\mathbf{u} \in W^{1, p}(\widehat{T})^{3}$ such that the right hand side of (5.2) vanishes while the left hand side does not. We will use the notation of Section 4 for the faces of $\widehat{T}$.

Proposition 5.1. For $k \geq 0$, the function $\mathbf{u}\left(x_{1}, x_{2}, x_{3}\right)=\left(x_{1}^{k+1}, 0,-(k+1) x_{1}^{k} x_{3}\right)$ satisfies $\operatorname{div} \mathbf{u}=0, u_{2}=0$ and $\Pi_{k, 2} \mathbf{u} \neq 0$.

Proof. We consider the case $k \geq 1$ (the case $k=0$ follows analogously). Since $\operatorname{div} \mathbf{u}=0$ we have $\Pi_{k, 1} \mathbf{u}, \Pi_{k, 3} \mathbf{u} \in \mathcal{P}_{k}(\widehat{T})$.

Now, using that $u_{1}=0$ on $\widehat{F}_{1}$ and $u_{3}=0$ on $\widehat{F}_{3}$ it follows from the definition of $\Pi_{k} \mathbf{u}$ that

$$
\int_{\widehat{F}_{1}} \Pi_{k, 1} \mathbf{u} p_{k}=0 \quad \forall p_{k} \in \mathcal{P}_{k}\left(\widehat{F}_{1}\right)
$$

and

$$
\int_{\widehat{F}_{3}} \Pi_{k, 3} \mathbf{u} p_{k}=0 \quad \forall p_{k} \in \mathcal{P}_{k}\left(\widehat{F}_{3}\right) .
$$

Then $\Pi_{k, 1} \mathbf{u}=x_{1} \alpha$ and $\Pi_{k, 3} \mathbf{u}=x_{3} \beta$ with $\alpha, \beta \in \mathcal{P}_{k-1}(\widehat{T})$. Also from the definition of $\Pi_{k} \mathbf{u}$ we have

$$
\int_{\widehat{F}_{2}}\left(\Pi_{k, 1} \mathbf{u}-\Pi_{k, 2} \mathbf{u}\right) p_{k}=\int_{\widehat{F}_{2}}\left(u_{1}-u_{2}\right) p_{k} \quad \forall p_{k} \in \mathcal{P}_{k}\left(\widehat{F}_{2}\right),
$$

and then, if $\Pi_{k, 2} \mathbf{u}=0$, we would obtain

$$
\int_{\widehat{F}_{2}} x_{1}\left(\alpha-x_{1}^{k}\right) p_{k}=0 \quad \forall p_{k} \in \mathcal{P}_{k}\left(\widehat{F}_{2}\right) .
$$

But taking $p_{k}=\alpha\left(x_{1}, x_{1}, x_{3}\right)-x_{1}^{k}$ in the last equation, we arrive at $\alpha\left(x_{1}, x_{1}, x_{3}\right)=$ $x_{1}^{k}$, but this contradicts the fact that $\alpha \in \mathcal{P}_{k-1}(\widehat{T})$. Then we have $\Pi_{k, 2} \mathbf{u} \neq 0$.

\section{ERROR ESTIMATES FOR RT INTERPOLATION}

We end the paper giving optimal error estimates for Raviart-Thomas interpolation of any order. These estimates are derived from the stability results obtained in the previous sections combined with polynomial approximation results.

Let us recall some well-known properties of the averaged Taylor polynomial. For a convex domain $D$ and any non-negative integer $m$, given $f \in W^{p, m+1}(D)$ the averaged Taylor polynomial is given by

$$
Q_{m} f(\mathbf{x})=\frac{1}{|D|} \int_{D} T_{m} f(\mathbf{y}, \mathbf{x}) d \mathbf{y}
$$

where

$$
T_{m} f(\mathbf{y}, \mathbf{x})=\sum_{|\alpha| \leq m} D^{\alpha} f(\mathbf{y}) \frac{(\mathbf{x}-\mathbf{y})^{\alpha}}{\alpha !} .
$$


Then, there exists a constant $C$, depending only on $m$ and $D$ (see for example [6, 13]), such that

$$
\left\|D^{\beta}\left(f-Q_{m} f\right)\right\|_{L^{p}(D)} \leq C \sum_{i_{1}+i_{2}+i_{3}=m+1}\left\|\frac{\partial^{m+1} f}{\partial x_{1}^{i_{1}} \partial x_{2}^{i_{2}} \partial x_{3}^{i_{3}}}\right\|_{L^{p}(D)}
$$

whenever $0 \leq|\beta| \leq m+1$.

As a consequence of these results we have the following approximation result for elements satisfying the regular vertex property. Given a function $f, D^{m} f$ denotes the sum of the absolute values of all the derivatives of order $m$ of $f$.

Lemma 6.1. Let $T$ be a tetrahedron satisfying $\operatorname{RVP}(\bar{c})$ such that $\mathbf{p}_{0}$ is the regular vertex, $\ell_{i}, i=1,2,3$ are unitary vectors with the directions of the edges sharing $\mathbf{p}_{0}$, $h_{i}, i=1,2,3$, the lengths of these edges, and $h_{T}$ the diameter of $T$. Then, given $\mathbf{u} \in W^{m+1, p}(T)^{3}, m \geq 0$, there exists $\mathbf{q} \in \mathcal{P}_{m}(T)^{3}$ such that,

$$
\left\|\frac{\partial(\mathbf{u}-\mathbf{q})}{\partial \ell_{1}}\right\|_{L^{p}(T)} \leq C \sum_{i_{1}+i_{2}+i_{3}=m} h_{1}^{i_{1}} h_{2}^{i_{2}} h_{3}^{i_{3}}\left\|\frac{\partial^{m+1} \mathbf{u}}{\partial \ell_{1}^{i_{1}+1} \partial \ell_{2}^{i_{2}} \partial \ell_{3}^{i_{3}}}\right\|_{L^{p}(T)}
$$

and analogously for $\frac{\partial(\mathbf{u}-\mathbf{q})}{\partial \ell_{j}}$ with $j=2,3$. Also,

$$
\|\operatorname{div}(\mathbf{u}-\mathbf{q})\|_{L^{p}(T)} \leq C h_{T}^{m}\left\|D^{m} \operatorname{div} \mathbf{u}\right\|_{L^{p}(T)},
$$

where the constant $C$ depends only on $m$ and $\bar{c}$.

Proof. To simplify notation we assume again that $\mathbf{p}_{0}=\mathbf{0}$. From Theorem 2.3 we know that there exists a matrix $M$ such that its associated linear transformation maps $\widetilde{T}$ onto $T$, where $\widetilde{T}$ is the element with vertices at $\mathbf{0}, h_{1} \mathbf{e}_{1}, h_{2} \mathbf{e}_{2}$ and $h_{3} \mathbf{e}_{3}$. Moreover, the norms of $M$ and of its inverse matrix are bounded by a constant which depends only on $\bar{c}$.

Now, as in the proof of Theorem 3.1, we define $\widetilde{\mathbf{u}} \in W^{m+1, p}(\widetilde{T})^{3}$ via the Piola transform and

$$
\widetilde{\mathbf{Q}}_{m} \widetilde{\mathbf{u}}=\left(\widetilde{Q}_{m} \tilde{u}_{1}, \widetilde{Q}_{m} \tilde{u}_{2}, \widetilde{Q}_{m} \tilde{u}_{3}\right) \in \mathcal{P}_{m}(\widetilde{T})^{3},
$$

where $\widetilde{Q}_{m} \tilde{u}_{j}$ denotes the averaged Taylor polynomial of $\tilde{u}_{j}$.

Using the estimate (6.1) on the reference element $\widehat{T}$ which has vertices at $\mathbf{0}, \mathbf{e}_{1}$, $\mathbf{e}_{2}$ and $\mathbf{e}_{3}$ and a standard scaling argument, we obtain

$$
\left\|\frac{\partial\left(\widetilde{\mathbf{u}}-\widetilde{\mathbf{Q}}_{m} \widetilde{\mathbf{u}}\right)}{\partial \tilde{x}_{1}}\right\|_{L^{p}(\widetilde{T})} \leq C \sum_{i_{1}+i_{2}+i_{3}=m} h_{1}^{i_{1}} h_{2}^{i_{2}} h_{3}^{i_{3}}\left\|\frac{\partial^{m+1} \widetilde{\mathbf{u}}}{\partial \tilde{x}_{1}^{i_{1}+1} \partial \tilde{x}_{2}^{i_{2}} \partial \tilde{x}_{3}^{i_{3}}}\right\|_{L^{p}(\widetilde{T})} .
$$

Then, defining $\mathbf{q} \in \mathcal{P}_{m}(T)^{3}$ via the Piola transform, that is,

$$
\mathbf{q}(\mathbf{x})=\frac{1}{\operatorname{det} M} M \widetilde{\mathbf{Q}}_{m} \widetilde{\mathbf{u}}(\widetilde{\mathbf{x}}), \quad \mathbf{x}=M \widetilde{\mathbf{x}},
$$

(6.2) follows by changing variables as in the proof of Theorem 3.1 .

On the other hand, since

$$
\widetilde{\operatorname{div}} \widetilde{\mathbf{Q}}_{m} \widetilde{\mathbf{u}}=\widetilde{Q}_{m-1} \widetilde{\operatorname{div}} \widetilde{\mathbf{u}},
$$

again using (6.1) in $\widehat{T}$ and a scaling argument, we obtain

$$
\left\|\widetilde{\operatorname{div}}\left(\widetilde{\mathbf{u}}-\widetilde{\mathbf{Q}}_{m} \widetilde{\mathbf{u}}\right)\right\|_{L^{p}(\widetilde{T})} \leq C h_{\widetilde{T}}^{m}\left\|\widetilde{D}^{m} \widetilde{\operatorname{div}} \mathbf{u}\right\|_{L^{p}(\widetilde{T})}
$$

and therefore, (6.3) follows by using the properties of the Piola transform stated in (3.9). 
We can now state and prove optimal error estimates for elements satisfying the regular vertex property. Our theorem generalizes the results proved in [1], where the same error estimate was proved in the case $k=0$, as well as those proved in [15, where the estimate was proved for any $k \geq 0$ but only in the case $m=k$.

Theorem 6.2. Let $k \geq 0$ and $T$ be a tetrahedron satisfying $\operatorname{RVP}(\bar{c})$. If $\mathbf{p}_{0}$ is the regular vertex, $\ell_{i}, i=1,2,3$ are unitary vectors with the directions of the edges sharing $\mathbf{p}_{0}, h_{i}, i=1,2,3$, the lengths of these edges, and $h_{T}$ the diameter of $T$, then there exists a constant $C$ depending only on $k$ and $\bar{c}$ such that, for $0 \leq m \leq k$, $1 \leq p \leq \infty$, and $\mathbf{u} \in W^{m+1, p}(T)^{3}$,

$$
\begin{aligned}
\| \mathbf{u} & -\Pi_{k} \mathbf{u} \|_{L^{p}(T)} \\
& \leq C\left\{\sum_{i_{1}+i_{2}+i_{3}=m+1} h_{1}^{i_{1}} h_{2}^{i_{2}} h_{3}^{i_{3}}\left\|\frac{\partial^{m+1} \mathbf{u}}{\partial \ell_{1}^{i_{1}} \partial \ell_{2}^{i_{2}} \partial \ell_{3}^{i_{3}}}\right\|_{L^{p}(T)}+h_{T}^{m+1}\left\|D^{m} \operatorname{div} \mathbf{u}\right\|_{L^{p}(T)}\right\} .
\end{aligned}
$$

Proof. Since $m \leq k$, for any $\mathbf{q} \in \mathcal{P}_{m}(T)^{3}$ we have

$$
\mathbf{u}-\Pi_{k} \mathbf{u}=\mathbf{u}-\mathbf{q}-\Pi_{k}(\mathbf{u}-\mathbf{q})
$$

and therefore, applying Theorem 3.1, we obtain

$$
\begin{aligned}
& \left\|\mathbf{u}-\Pi_{k} \mathbf{u}\right\|_{L^{p}(T)} \\
& \leq C\left\{\|\mathbf{u}-\mathbf{q}\|_{L^{p}(T)}+\sum_{i, j} h_{j}\left\|\frac{\partial\left(u_{i}-q_{i}\right)}{\partial \ell_{j}}\right\|_{L^{p}(T)}+h_{T}\|\operatorname{div}(\mathbf{u}-\mathbf{q})\|_{L^{p}(T)}\right\} .
\end{aligned}
$$

Then, taking $\mathbf{q} \in \mathcal{P}_{m}(T)^{3}$ satisfying (6.2) and (6.3) we conclude the proof.

Optimal error estimates under the maximum angle condition can also be proved. We state the results in the following theorem.

Theorem 6.3. Let $k \geq 0$ and $T$ be a tetrahedron with diameter $h_{T}$ satisfying $\operatorname{MAC}(\bar{\psi})$. There exists a constant $C$ depending only on $k$ and $\bar{\psi}$ such that, for $0 \leq m \leq k, 1 \leq p \leq \infty$, and $\mathbf{u} \in W^{m+1, p}(T)^{3}$,

$$
\left\|\mathbf{u}-\Pi_{k} \mathbf{u}\right\|_{L^{p}(T)} \leq C h_{T}^{m+1}\left\|D^{m+1} \mathbf{u}\right\|_{L^{p}(T)} .
$$

The proof is analogous to that of the previous theorem, now using the stability estimates obtained in Theorem 4.1, and so we omit the details.

\section{REFERENCES}

[1] G. Acosta, R. G. DurÁn, The maximum angle condition for mixed and nonconforming elements: Application to the Stokes equations, SIAM J. Numer. Anal. 37, 18-36, 1999. MR.1721268 (2000g:65107)

[2] T. APEL, Anisotropic Finite Elements: Local Estimates and Applications, Teubner, Stuttgart, 1999. MR.1716824 (2000k:65002)

[3] D. N. ARNold, F. BrezzI, Mixed and nonconforming finite element methods: implementation, postprocessing and error estimates, RAIRO Modél. Math. Anal. Numer. 19, 7-32, 1985. MR813687 (87g:65126)

[4] I. BABuska, A. K. AzIz, On the angle condition in the finite element method, SIAM J. Numer. Anal. 13, 214-226, 1976. MR0455462 (56:13700)

[5] A. Bermúdez, R. Durán, M. A. Muschietti, R. Rodríguez, J. Solomin, Finite element vibration analysis of fluid-solid systems without spurious modes, SIAM J. Numer. Anal. 32, 1280-1295, 1995. MR.1342293 (96e:73072)

[6] S. Brenner, L. R. Scott, The Mathematical Analysis of Finite Element Methods, Springer Verlag, 1994. MR1278258 (95f:65001) 
[7] F. Brezzi, M. Fortin, Mixed and Hybrid Finite Element Methods, Springer Verlag, 1991. MR 1115205 (92d:65187)

[8] F. Brezzi, M. Fortin And R. Stenberg, Error analysis of mixed-interpolated elements for Reissner-Mindlin plates, Math. Models and Methods in Appl. Sci. 1, 125-151, 1991. MR:1115287 (92e:73030)

[9] A. Buffa, M. Costabel, M. Dauge, Algebraic convergence for anisotropic edge elements in polyhedral domains, Numer. Math. 101, 29-65, 2005. MR2194717 (2006j:65339)

[10] P. G. Ciarlet, The Finite Element Method for Elliptic Problems, North-Holland, 1978. MR0520174 (58:25001)

[11] J. Douglas, J. E. Roberts, Global estimates for mixed methods for second order elliptic equations, Math. Comp. 44, 39-52, 1985. MR771029 (86b:65122)

[12] R. G. DuRÁn, Error estimates for anisotropic finite elements and applications, Proceedings of the International Congress of Mathematicians, Madrid, 2006, Volume III, pp. 1181-1200. MR2275724 (2008c:65321)

[13] R. G. Durán, Mixed Finite Element Methods, in Mixed Finite Elements, Compatibility Conditions, and Applications, D. Boffi and L. Gastaldi, eds., Lecture Notes in Mathematics 1939, Springer, 1-44, 2008. MR2459075

[14] R. G. Durán, E. Liberman, On Mixed Finite Element Methods for the Reissner Mindlin Plate Model, Math. Comp. 58, 561-573, 1992. MR.1106965 (92f:65135)

[15] R. G. Durán, A. L. Lombardi, Error estimates for the Raviart-Thomas interpolation under the maximum angle condition, SIAM J. Numer. Anal., 46, 1442-1453, 2008. MR2391001 (2009b:65302)

[16] M. Farhloul, S. Nicaise and L. Paquet, Some mixed finite element methods on anisotropic meshes, Math. Modeling Numer. Anal. 35, 907-920, 2001. MR1866274 (2002h:65181)

[17] P. JAMET, Estimations d'erreur pour des éléments finis droits presque dégénérés, RAIRO Anal. Numérique 10, 43-60, 1976. MR0455282 (56:13521)

[18] M. KŘÍŽEK, On the maximum angle condition for linear tetrahedral elements, SIAM J. Numer. Anal. 29, 513-520, 1992. MR.1154279 (92k:65165)

[19] L. D. MARINI, An inexpensive method for the evaluation of the solution of the lowest order Raviart-Thomas mixed method, SIAM J. Numer. Anal. 22, 493-496, 1985. MR787572 (86g:65214)

[20] J.-C. NÉDÉLEC, Mixed finite elements in $\mathbb{R}^{3}$, Numer. Math. 35, 315-341, 1980. MR592160 (81k:65125)

[21] P. A. Raviart, J.-M. Thomas, A mixed finite element method for second order elliptic problems, in Mathematical Aspects of Finite Element Methods, I. Galligani, E. Magenes, eds., Lectures Notes in Math. 606, Springer Verlag, 1977. MR0483555 (58:3547)

[22] J. L. Synge, The hypercircle in mathematical physics, Cambridge University Press, Cambridge, 1957. MR0097605 (20:4073)

[23] J.-M. Thomas, Sur l'analyse numérique des méthodes d'éléments finis hybrides et mixtes, Thèse d'Etat, Université Pierre et Marie Curie, Paris, 1977.

Departamento de Matemática, Facultad de Ciencias Exactas y Naturales, Universidad de Buenos Aires, 1428 Buenos Aires, Argentina. Member of COniCet, Argentina.

E-mail address: gacosta@dm.uba.ar

Institut für Mathematik und Bauinformatik, Universität der Bundeswehr MÜnchen, Neubiberg, Germany.

E-mail address: thomas.apel@unibw.de

Departamento de Matemática, Facultad de Ciencias Exactas y naturales, Universidad de Buenos Aires, 1428 Buenos Aires, Argentina. Member of COniCEt, Argentina.

E-mail address: rduran@dm.uba.ar

Instituto de Ciencias, Universidad Nacional de General Sarmiento, J.M. Gutierrez 1150, Los Polvorines, B1613GSX Provincia de Buenos Aires, Argentina and Departamento de Matemática, Facultad de Ciencias Exactas y Naturales, Universidad de Buenos Aires, 1428 Buenos Aires, Argentina. Member of COniCEt, Argentina.

E-mail address: aldoc7@dm.uba.ar 